\title{
Killing Tensors and the Separation of the Hamilton-Jacobi Equation *
}

\author{
N. M. J. Woodhouse \\ Department of Mathematics, University of London, King's College, London, U.K.
}

Received May 25, 1974

\begin{abstract}
This paper investigates the relationship between Killing Tensors and separable systems for the geodesic Hamilton-Jacobi equation in Riemannian and Lorentzian manifolds: locally, a separable system consists of the vector and covector associated with a separable coordinate. It is shown that there are only two types of separable system, those associated with local symmetry groups and those which can be obtained by a simple transformation from orthogonal systems. Some sufficient conditions for existence are given and some global problems are enumerated. The results are illustrated with a demonstration that the existence of separable systems in a certain class of $\{2,2\}$ space-times is a consequence of the algebraic properties of the Weyl tensor.
\end{abstract}

\section{§ 1. Introduction}

During the century or so following the work of Jacobi, much research in Hamiltonian mechanics was directed towards understanding the separability conditions for the Hamilton-Jacobi equation; this is not surprising since the Hamilton-Jacobi method was (and still is) one of the few analytical tools available. But, with the shift in emphasis in classical mechanics from the local and quantitative to the global and qualitative, interest in this line of research waned, only recently to be revived, first, in general relativity, as a result of Carter's observation that the Hamilton-Jacobi equation for the geodesics in the Kerr solution can be solved by separating the variables [5] and of the subsequent exploitation of this fact in astrophysical calculations (for example, by Bardeen [2]) and, secondly, in quantum mechanics, through the investigation of its relationship to degeneracy and dynamical symmetry in the context of intrinsic quantization (for example, Onofri and Pauri [21]).

The classical research, initiated by Liouville [18] and Stäckel [25] and culminating in a paper by Iarov-Iarovoi [14] in 1964, was aimed, in part, at finding the most general coordinate form of a metric and potential with a separable Hamilton-Jacobi equation. Unfortunately, the use of local "arithmetical" methods did not lead to any real geometrical insights. For example, one feature of the simpler classical and relativistic systems, which is still not properly understood, is that the constants of the motion which arise from the local separation of the Hamilton-Jacobi equation are well behaved in the large. It is likely that this is related to other separability properties of these systems (for instance, to the separability of the associated Schrödinger equations) since the separation of a

$\star$ Research supported by the Science Research Council. 
wave equation is also an essentially global property (thus, the fact that Laplace's equation is separable in Euclidean space in spherical polar coordinates depends not only on the naïve observation that the substitution of a particular form for the wave function leads to consistent separated equations but also on the fact that a certain complete subset of the solutions constructed in this way are well behaved in the large). Recently, Onofri and Pauri have made some progress in the understanding of global properties of Hamilton-Jacobi separable systems through their research into the general problems of intrinsic quantization [21]. It is hoped that the present investigation, which is essentially orthogonal to theirs, will result in greater geometrical insight into the role of separability in the specific problem of finding particle orbits in Lorentzian and Riemannian manifolds and, ultimately, to a understanding of the relationship between this and other separability properties.

The present paper deals only with the free particle (geodesic) case; subsequent papers will deal with potentials and, it is hoped, with the wider questions. Specifically, the bulk of this paper investigates, locally and, to some extent, globally, what are called separable systems for the geodesics in a Riemannian manifold: locally, a separable system consists of the vector and covector associated with a coordinate which can be separated in the Hamilton-Jacobi equation. By concentrating on a single coordinate, I hoped to achieve a geometrical formalism better suited to the investigation of global problems than the classical approach of analysing entire separable coordinate systems. A fact which emerges clearly from this approach, and which was not over-emphasized in the past, is that it is not actually necessary to solve the Hamilton-Jacobi equation in order to find the constants of the motion. For instance, if it is known that just one coordinate in the Hamilton-Jacobi equation is separable it is possible to write down, by inspecting the contravariant form of the metric, a number of linear or quadratic constants of the motion for the geodesics ${ }^{1}$. In the case of the $\theta$ coordinate in the Kerr solution, a complete set can be constructed in this way.

The Kerr solution has a number of special features; prominent among these are the algebraic type of its Weyl tensor (through which the solution was discovered in the first place [15]) and its separability properties: in addition to the geodesic Hamilton-Jacobi equation, the spin-zero wave equation [5] and a certain decoupled part of the perturbed Bianchi identity (the Teukolsky equation [30]) are both separable in the standard Boyer-Lindquist coordinate system [3]. An investigation into the puzzling relationship between these features has been initiated by Walker, Penrose, Hughston and Sommers [13, 28, 29, 31]. This paper fits into their line of research in that one of its applications is a proof that the existence of the three independent Killing tensors, which can be inferred from the algebraic classification of the Weyl tensor $[13,31]$, implies the separability of the Hamilton-Jacobi equation (the converse of this being the essential content of Carter's work [5]). The motivation here is not simply mathematical curiosity;

1 Unfortunately, the more constants of the motion can be constructed in this way, the harder it is to spot that the coordinate is, in fact, separable. However a procedure that might be practical is this: by use of a little inspired guesswork, pick a likely candidate for a separable coordinate and write down what would be the constants of the motion if the coordinate were separable and then check that the corresponding tensors (see $\S 3$ ) are in fact Killing tensors. 
there remain practical problems which are unlikely to be solved without a deeper understanding of this relationship. For example, the completeness of the normal modes of the Teukolsky equation (which is important in the investigation of the stability of the solution [30]) has yet to be rigorously established [32].

Throughout, I shall use coordinate free methods. The notation of the first five sections is explained below. In the sixth section, which should be regarded as an appendix, I apply some of my results to type $\{2,2\}$ spacetimes; here a slightly different notation is used which is better suited to detailed spinor calculations (the Battelle convention [22]).

A rough plan of the other sections is as follows: $\S 2$ is a brief modern treatment of Hamilton-Jacobi theory; this owes much to Hermann [12]. §3 discusses Killing tensors and some generalizations. $\S 4$ investigates the local relationship between separability and Killing tensors, while $\S 5$ discusses some of the problems involved in globalizing the results.

\section{Notation}

All differentiable structures are smooth (class $C^{\infty}$ ).

The ring of smooth real valued functions on a manifold $M$ is denoted by $F(M) ; S^{q}(M)$ is the $F(M)$ module of symmetric contravariant tensor fields of valence $q$ and $S(M)$ is the union $\bigcup_{q \geqq 0} S^{q}(M)$. The intersection symbol $\cap$ is used to denote symmetrized tensor multiplication in $S(M)$ (after Sommers [27]). The module of exterior $q$-forms is represented by $\wedge_{q}(M)$ and, in particular, $\wedge_{0}(M)=$ $S^{0}(M)=F(M)$.

The cotangent bundle of $M$ is written:

$$
\Pi: T^{*} M \rightarrow M
$$

and the tangent and cotangent spaces at $m \in M$ are denoted by $T_{m} M$ and $T_{m}^{*} M$ respectively.

A tensor field (or an exterior form) on a manifold plays a large number of different roles. For example, $A \in S^{q}(M)$ is a multilinear map from $\wedge_{1}(M) \times \wedge_{1}(M) \times \ldots$ $\wedge_{1}(M)$ to $F(M)$, a linear map from $\wedge_{1}(M)$ to $S^{q-1}(M)$, a real function on $T^{*} M$, a tensor over the real vector space $T_{m} M$ for each $m \in M$, a cross-section of a vector bundle over $M$ and so on. In general, there is no need to use different symbols for the same tensor when it is acting in different roles and this will not be done: the role is obvious from the argument. However, in the interests of clarity, three exceptions will be made:

1) For emphasis, the value of a tensor field $T$ at some point $m \in M$ will occasionally (but not always) be distinguished from $T$ itself by use of the symbol $T_{(m)}$.

2) A 1-form on $M$ is both an algebraic object (an element of the $F(M)$ module $\wedge_{1}(M)$ ) and a geometric object (a cross-section of $T^{*} M$ ). The distinction between these two objects (which is purely notational) will be emphasized by denoting the geometric object by the same Greek letter as the algebraic object, but in capital script. Thus if $\varphi \in \wedge_{1}(M)$ then $\varphi: S^{1}(M) \rightarrow F(M)$ while $\Phi: M \rightarrow T^{*} M$, $\Phi^{*}: \wedge_{1}\left(T^{*} M\right) \rightarrow \wedge_{1}(M)$ and so on.

3) Each tensor in $S(M)$ defines a real function on $T^{*} M$; this function will be represented by the same Roman letter as the tensor, but in the lower case. Thus 
if $A \in S^{q}(M)$ then $a \in F\left(T^{*} M\right)$ is given by:

$$
\begin{aligned}
& a=A \circ \Pi ; \quad q=0, \\
& a:(m, \boldsymbol{p}) \mapsto A_{(m)}(\boldsymbol{p}, \boldsymbol{p} \ldots \boldsymbol{p}) \in \mathbb{R} ; \quad q>0, \quad(m, \boldsymbol{p}) \in T^{*} M .
\end{aligned}
$$

Both these last two conventions are consistent with the following usage: tensors and vectors in $S(M)$ are denoted by capital italic letters, real numbers and real valued functions by lower case italic letters, exterior forms by lower case Greek letters and maps of manifolds by capital Greek letters. Covariant vectors and tensors will be printed in bold faced type.

For reference, and to fix sign conventions, I collect together some elementary definitions and results; proofs may be found in Loomis and Sternberg [19] or in Abraham [1]:

The canonical 1 -form $\theta \in \wedge_{1}\left(T^{*} M\right)$ is defined by:

$$
\theta_{(m, \boldsymbol{p})}(X)=\boldsymbol{p}\left(\Pi_{*}(X)\right) ; \quad(m, \boldsymbol{p}) \in T^{*} M, \quad X \in T_{(m, \boldsymbol{p})} T^{*} M .
$$

The canonical 2-form is the exterior derivative $\omega=d \theta ; \omega$ is a symplectic structure on $T^{*} M$. Each 1-form $\alpha \in \wedge_{1}\left(T^{*} M\right)$ is associated with a vector $\alpha^{\#} \in S^{1}(M)$ given by:

$$
\left.\alpha^{\#}\right\rfloor \omega+\alpha=0
$$

where the contraction $\downarrow$ is defined by:

$$
(A\lrcorner \beta)=2 \beta(A, B) ; \quad A, B \in S^{1}(M), \quad \beta \in \wedge_{2}(M) .
$$

The Poisson bracket of two functions on $T^{*} M$ is defined by:

$$
[a, b]=2 \omega\left(d a^{\#}, d b^{\#}\right) \in F\left(T^{*} M\right) ; \quad a, b \in F\left(T^{*} M\right) .
$$

The Poisson bracket gives $F\left(T^{*} M\right)$ the structure of an infinite dimensional Lie algebra over $\mathbb{R}$.

The Lie derivative [with respect to $T \in S^{1}(M)$ ] is written $\mathscr{L}_{T}$; it is related to the exterior derivative by:

$$
\left.\begin{array}{rl}
2 d \alpha(A, B) & =A(\alpha(B))-B(\alpha(A))-\alpha\left(\mathscr{L}_{A} B\right) \\
d(\alpha(A)) & =\mathscr{L}_{A} \alpha-A \downarrow d \alpha
\end{array}\right\} \alpha \in \wedge_{1}(M), \quad A, B \in S^{1}(M)
$$

and to the Poisson bracket by:

$$
(d([a, b]))^{\#}=-\left[d a^{\#}, d b^{\#}\right]=-\mathscr{L}_{d a^{\#}}\left(d b^{\#}\right) ; \quad a, b \in F\left(T^{*} M\right) .
$$

Also, if $A, B \in S^{1}(M)$ and if $a, b: T^{*} M \rightarrow \mathbb{R}$ are the corresponding functions in $F\left(T^{*} M\right)$ then:

$$
[a, b](m, \boldsymbol{p})=-\boldsymbol{p}\left(\mathscr{L}_{A} B\right) ; \quad(m, \boldsymbol{p}) \in T^{*} M .
$$

Finally, a vector $X \in T_{(m, \boldsymbol{p})} T^{*} M,(m, \boldsymbol{p}) \in T^{*} M$, is said to be vertical if $\Pi_{*}(X)=0$; if $X, Y \in T_{(m, \boldsymbol{p})} T^{*} M$ are vertical then:

$$
\begin{aligned}
\theta(X) & =0, \\
\omega(X, Y) & =0 .
\end{aligned}
$$




\section{§ 2. Hamilton-Jacobi Theory}

A conservative Hamiltonian system consists of a configuration space $M$ (a smooth $n$-dimensional manifold), a phase space $T^{*} M$ (the cotangent bundle of $M$ ) and a Hamiltonian $h: T^{*} M \rightarrow \mathbb{R}$. The orbits of the system in $T^{*} M$ are the integral curves of the vector field $X_{h}=d h^{\#}$ defined by Hamilton's equation:

$$
\left.X_{h}\right\lrcorner \omega+d h=0 .
$$

A classical approach to the problem of finding an analytical description of the orbits and their projections in configuration space is to look for constants of the motion, that is for functions $c: T^{*} M \rightarrow \mathbb{R}$ which commute with $h$ :

$$
[c, h]=0 .
$$

Such functions are first integrals of the motion (their level surfaces are tangent to $\left.X_{h}\right)$; together, they form a Lie subalgebra of $F\left(T^{*} M\right)$ since if $c_{1}$ and $c_{2}$ commute with $h$, then so do $c_{1} \cdot c_{2},\left[c_{1}, c_{2}\right]$ and $r \cdot c_{1}+c_{2}$ (where $r \in \mathbb{R}$ ). Locally, any Hamiltonian system admits $2 n-1$ functionally independent constants of the motion though, in general, these cannot be extended over the whole of $T^{*} M$ : in the large, their level surfaces will not form invariant submanifolds of $T^{*} M$ [8] (that is submanifolds made up of orbits of $X_{h}$ ).

According to the ancient technique, local constants of the motion can be constructed from solutions of the Hamilton-Jacobi partial differential equation. The way in which this works is as follows: let $\varphi \in \Lambda_{1}(M)$ be a 1-form (thought of as an algebraic object) and let $\Phi: M \rightarrow T^{*} M$ be the corresponding cross-section of $T^{*} M$ (this notation is explained in $\S 1$ ). With the observation that

$$
\Pi_{*} \circ \Phi_{*}: S^{1}(M) \rightarrow S^{1}(M)
$$

is the identity, one may establish the basic lemmas:

2.1. Proposition. $\Phi^{*} \theta=\varphi(\theta$ is the canonical 1-form).

Proof. If $X \in T_{m} M, m \in M$, then $\Phi_{*} X$ is a tangent vector to $T^{*} M$ at $\left(m, \varphi_{(m)}\right) \in$ $\Phi(M)$ and:

$$
\left(\Phi^{*} \theta\right)(X)=\theta\left(\Phi_{*} X\right)=\varphi\left(\Pi_{*}\left(\Phi_{*} X\right)\right)=\varphi(X) .
$$

2.2. Proposition. If $X$ is a tangent vector to $T^{*} M$ at $(m, \boldsymbol{p}) \in \Phi(M)$ then $\Phi_{*}\left(\Pi_{*} X\right)-X$ is vertical.

Proof. A direct calculation gives:

$$
\Pi_{*}\left(\Phi_{*}\left(\Pi_{*} X\right)-X\right)=\Pi_{*} X-\Pi_{*} X=0 \text {. }
$$

2.3. Proposition. If $X$ and $Y$ are tangent vectors to $T^{*} M$ at some point $(m, \boldsymbol{p}) \in \Phi(M) \subset T^{*} M$ then:

1) $\omega\left(\Phi_{*}\left(\Pi_{*} X\right), \Phi_{*}\left(\Pi_{*} Y\right)\right)=d \varphi\left(\Pi_{*} X, \Pi_{*} Y\right)$,

2) $\omega\left(\Phi_{*}\left(\Pi_{*} X\right), Y\right)+\omega\left(X, \Phi_{*}\left(\Pi_{*} Y\right)\right)=\omega(X, Y)+d \varphi\left(\Pi_{*} X, \Pi_{*} Y\right)$

(here $\omega$ is the canonical 2-form). 
Proof. The first part follows from the observation:

$$
\omega\left(\Phi_{*}\left(\Pi_{*} X\right), \Phi_{*}\left(\Pi_{*} Y\right)\right)=\left(\Phi^{*} \omega\right)\left(\Pi_{*} X, \Pi_{*} Y\right)
$$

together with the corollary of Proposition 2.1:

$$
\Phi^{*} \omega=\Phi^{*}(d \theta)=d\left(\Phi^{*} \theta\right)=d \varphi .
$$

The second part follows by expanding:

$$
\omega\left(\Phi_{*}\left(\Pi_{*} X\right)-X, \Phi_{*}\left(\Pi_{*} Y\right)-Y\right)
$$

which is zero by Proposition 2.2 and Eq. (1.12).

2.4. Proposition. If $\varphi$ is closed and if $a, b: T^{*} M \rightarrow \mathbb{R}$ are two functions such that: $a \circ \Phi=$ const ; $\quad b \circ \Phi=$ const then $[a, b]$ vanishes on $\Phi(M)$.

Proof. Put $X=d a^{\#}$ and $Y=d b^{\#}$ and, using Proposition 2.3, evaluate $\omega(X, Y)=$ $\frac{1}{2}[a, b]$ on $\Phi(M)$; the result is zero since $d \varphi=0$ and, at points of $\Phi(M)$ :

$$
\begin{aligned}
\omega\left(\Phi_{*}\left(\Pi_{*} X\right), Y\right) & =\Phi_{*}\left(\Pi_{*} X\right)(b)=\left(\Pi_{*} X\right)(b \circ \Phi) \\
& =0 \\
& =\omega\left(X, \Phi_{*}\left(\Pi_{*} Y\right)\right) .
\end{aligned}
$$

A closed 1-form $\varphi$ is said to be a solution of the Hamilton-Jacobi equation if the corresponding cross-section $\Phi: M \rightarrow T^{*} M$ satisfies:

$h \circ \Phi=$ const .

Locally, $\varphi=d s$ for some real function $s \in F(M)$ and, in coordinates $\left\{x^{a}\right\}$, the Hamilton-Jacobi equation assumes its familiar form:

$$
h\left(x^{\mathfrak{a}}, \frac{\partial s}{\partial x^{\mathfrak{a}}}\right)=\text { const } .
$$

A local complete solution of the Hamilton-Jacobi equation is a local trivialization $\Psi: U \times V \subset M \times \mathbb{R}^{n} \rightarrow W \subset T^{*} M$ of $T^{*} M$ such that, for each $v \in V$, the restriction $\Psi_{v}: U \rightarrow T^{*} M$ defines a closed 1-form $\psi_{v} \in \wedge_{1}(M)$ which satisfies the HamiltonJacobi equation ( $U \subset M, W \subset \Pi^{-1}(U)$ and $V \subset \mathbb{R}^{n}$ are open sets). Essentially, a local complete solution is an $n$-parameter family of solutions of the Hamilton-Jacobi equation.

Putting $b=h$ in Proposition 2.4, one obtains:

2.5. Proposition. If $\varphi$ is a solution of the Hamilton-Jacobi equation then $X_{h}$ is tangent to $\Phi(M)$.

Proof. On $\Phi(M), X_{h}(a)=-[a, h]=0$ for any function $a \in F\left(T^{*} M\right)$ such that $\left.a\right|_{\Phi(M)}$ is constant: this implies that $X_{h}$ is tangent to $\Phi(M)$.

The key result of Hamilton-Jacobi theory is that any function of the parameters in a complete solution is a local constant of the motion; stated precisely:

2.1. Theorem (Hamilton-Jacobi theorem). Let $\Psi: U \times V \subset M \times \mathbb{R}^{n} \rightarrow T^{*} M$ be a local complete solution of the Hamilton-Jacobi equation and let $\Pi_{2}$ denote the 
projection:

$$
\Pi_{2}: U \times V \rightarrow V .
$$

If $k: V \rightarrow \mathbb{R}$ is any smooth function then:

$$
c=k \circ \Pi_{2} \circ \Psi^{-1}: T^{*} M \rightarrow \mathbb{R}
$$

is a local constant of the motion.

Proof. The theorem is a corollary of Proposition 2.5 since if $X_{h}$ is tangent to $\Psi_{v}(U) \subset T^{*} M$ for each $v \in V$ then it must also be tangent to $c^{-1}(t)$ for each $t \in \mathbb{R}$.

Remarks. 1) If $c_{1}$ and $c_{2}$ are two constants of the motion constructed in this way from a complete solution $\Psi$ then $\left[c_{1}, c_{2}\right]=0$. This follows from Proposition 2.5 since $c_{1}$ and $c_{2}$ are constant on each section $\Psi_{v}(U) \subset T^{*} M$ of $T^{*} M$. Put another way, $\Psi$ defines a local foliation of $T^{*} M$ by Lagrangian submanifolds, that is a local polarization of $T^{*} M[16]$.

2) A set $\left\{a_{1}, a_{2} \ldots a_{m}\right\} \subset F\left(T^{*} M\right)$ of functions on $T^{*} M$ will be said to be vertically independent if no linear combination (over $F\left(T^{*} M\right)$ ) of the vector fields $d a_{1}^{\#}, d a_{2}^{\#}, \ldots d a_{m}^{\#}$ is vertical (alternatively, if the restrictions of $a_{1}, a_{2} \ldots a_{m}$ to any fibre of $T^{*} M$ are functionally independent). By the inverse function theorem, any set of $n$ vertically independent commuting constants of the motion $\left\{c_{1}, c_{2} \ldots c_{n}\right\}$ defines a local complete solution [closure follows by putting $X=d c_{i}^{\#}, Y=d c_{j}^{\#}$, $i, j=1,2 \ldots n$, and using the first part of Proposition 2.3 together with the fact that $\Pi_{*}\left(d c_{1}^{\#}\right), \Pi_{*}\left(d c_{2}^{\#}\right) \ldots \Pi_{*}\left(d c_{n}^{\#}\right)$ are linearly independent over $\left.F(M)\right]$. The converse of this remark is an immediate corollary of Theorem 2.1.

3) It is often interesting (or merely practical) to look for the orbits only on a single energy surface in $T^{*} M$. The Hamilton-Jacobi method has a simple generalization in this case: a complete local solution of the Hamilton-Jacobi equation for the energy surface $N=h^{-1}(t) \subset T^{*} M$ is a local diffeomorphism $\Psi: U \times V \subset$ $M \times \mathbb{R}^{n-1} \rightarrow \Pi^{-1}(U) \cap N \subset T^{*} M$ such that each restriction $\Psi_{v}: U \rightarrow T^{*} M$ defines a (local) closed solution of the Hamilton-Jacobi equation ( $U \subset M$ and $V \subset \mathbb{R}^{n-1}$ are open sets). Functions of the form $c=k \circ \Pi_{2} \circ \Psi^{-1}$, where $k: V \rightarrow \mathbb{R}$, are constants of the motion for the orbits in $N$. A particular example is the problem of finding the null geodesics in a pseudo-Riemannian manifold: a complete local solution for the null energy surface will be called a complete null solution.

In the remainder of this paper, I shall be concerned with the Hamiltonian:

$$
h:(m, \boldsymbol{p}) \mapsto \frac{1}{2} G(\boldsymbol{p}, \boldsymbol{p})
$$

for the geodesics in a Riemannian or pseudo-Riemannian manifold $(M, g)$ $\left[G \in S^{2}(M)\right.$ is the inverse contravariant metric]. A subsequent paper will deal with potentials.

\section{§ 3. Killing Tensors and Some Generalizations}

The Lie bracket operation for vector fields on a manifold $M$ extends naturally to a skew product on the set $S(M)$ of symmetric contravariant tensor fields on $M$ [20]. If $A \in S^{p}(M)$ and $B \in S^{q}(M)$ then the product, $[A, B]$, is an element of 
$S^{p+q-1}(M)$. In the simplest cases, $p=q=0$ and $p=1,[A, B]$ is given by:

$$
\begin{aligned}
& {[A, B]=0 ; \quad A, B \in S^{0}(M),} \\
& {[A, B]=\mathscr{L}_{A} B ; \quad A \in S^{1}(M), \quad B \in S(M) .}
\end{aligned}
$$

When $p>0$ and $A$ has a decomposition of the form:

$$
A=X_{1} \cap X_{2} \cap \ldots \cap X_{p} ; \quad X_{i} \in S^{-1}(M)
$$

the product is defined by:

$$
\begin{aligned}
{[A, B] } & =X_{1} \cap X_{2} \cap \ldots \cap X_{p-1} \cap\left[X_{p}, B\right] \\
& +\ldots+\left[X_{1}, B\right] \cap X_{2} \cap \ldots \cap X_{p} .
\end{aligned}
$$

In the general case, $[\mathrm{A}, \mathrm{B}]$ is constructed by writing:

$$
\mathrm{A}=\sum_{i} \mathrm{~A}_{i}
$$

where each $A_{i}$ is decomposable as in Eq. (3.3), and putting:

$$
[A, B]=\sum_{i}\left[A_{i}, B\right] \text {. }
$$

It is not hard to see that this definition is independent of the precise way in which $A$ is decomposed and that the resulting product is skew symmetric. Also it can be established by straightforward inductive arguments that the product obeys the distributive law and the Leibnitz rule:

$$
\begin{aligned}
{[A+r B, C] } & =[A, C]+r[B, C] ; \quad A, B \in S^{p}(M), \quad C \in S^{q}(M), \quad r \in \mathbb{R}, \\
{[A \cap B, C] } & =[A, C] \cap B+A \cap[B, C] ; \quad A, B, C \in S(M),
\end{aligned}
$$

that it satisfies the Jacobi identity:

$$
[A,[B, C]]+[B,[C, A]]+[C,[A, B]]=0
$$

and that, in local coordinates, it has components:

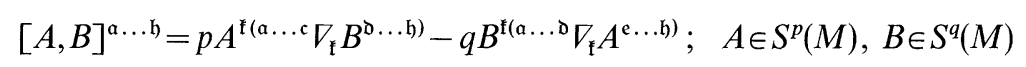

where $\nabla$ is any torsion free linear connexion on $M$.

Looked at in another way, the skew product on $S(M)$ is essentially the same as the Poisson bracket on $F\left(T^{*} M\right)$. Explicitly, each tensor $A \in S(M)$ is associated with a real function $a: T^{*} M \rightarrow \mathbb{R}$ as in Eqs. (1.2) and (1.3). In the following, the function in $F\left(T^{*} M\right)$ corresponding to a tensor in $S(M)$ will be denoted by the same letter as the tensor, but in the lower case; with this convention:

3.1. Proposition. Let $A, B \in S(M)$. If $C=[A, B]$ then $c=-[a, b]$.

The proof is either by induction [the proposition reduces to Eq. (1.10) when $\left.A, B \in S^{1}(M)\right]$ or by direct computation, using Eq. (3.10).

In fact, $S(M)$ can be identified with a certain Lie subalgebra of $F\left(T^{*} M\right)$. To be precise, let $\bar{S}(M)$ denote the real vector subspace of $\bigoplus_{p \geqq 0} S^{p}(M)$ made up of finite formal sums:

$$
A_{0}+A_{1}+\ldots+A_{q} ; \quad A_{i} \in S^{i}(M)
$$


and let $\bar{F}\left(T^{*} M\right)$ be the subspace of $F\left(T^{*} M\right)$ consisting of functions on $T^{*} M$ which are polynomial in $\boldsymbol{p}$. The skew product defined by Eqs. (3.1)-(3.5) extends to give $\bar{S}(M)$ the structure of an infinite dimensional Lie algebra over $\mathbb{R}$ and the map $i: \bar{S}(M) \rightarrow \bar{F}\left(T^{*} M\right)$ given by:

$$
i\left(A_{0}+A_{1}+\ldots+A_{q}\right)=a_{0}+a_{1}+\ldots+a_{q} \in \bar{F}\left(T^{*} M\right)
$$

defines a Lie algebra isomorphism between $\bar{S}(M)$ and $\bar{F}\left(T^{*} M\right)$.

The symmetric tensor product $\cap$ also extends to $\bar{S}(M)$, so that $\bar{S}(M)$ has one further structure: with respect to $\cap$ and,$+ \bar{S}(M)$ forms a ring (and, in fact, an algebra over $\mathbb{R}$ ); it is simply a polynomial ring of $F(M)$. The relationship between these two algebraic structures on $\bar{S}(M)$ can be clarified by remarking that the adjoint representation of its Lie algebra structure embeds $\bar{S}(M)$ in the Lie algebra of derivations [26] of $(\bar{S}(M), \cap,+)$.

Let $\boldsymbol{g}$ be a Riemannian or pseudo-Riemannian metric on $M$. A Killing tensor [24] on $(M, g)$ is a tensor field $K \in S(M)$ which satisfies:

$$
[K, G]=0
$$

where $G \in S^{2}(M)$ is the inverse contravariant metric. In local coordinates, this equation can be written in either of the two forms:

$$
\begin{aligned}
& q K^{\mathfrak{f}(\mathfrak{a} \ldots \mathrm{d}} \partial_{\mathfrak{f}} G^{\mathfrak{e})}-2 G^{\mathfrak{f}(\mathfrak{a}} \partial_{\mathfrak{f}} K^{\mathfrak{b} \ldots \mathfrak{f})}=0 ; \quad K \in S^{q}(M), \\
& \nabla^{(\mathfrak{a}} K^{\mathfrak{b} \ldots \mathfrak{f})}=0 .
\end{aligned}
$$

Here $\partial$ is the coordinate partial derivative and $\nabla$ is the metric connexion. Note that the only Killing tensors of valence zero are constants.

If $K_{1}$ and $K_{2}$ are Killing tensors then so are $c K_{1}+K_{2}(c \in \mathbb{R}), K_{1} \cap K_{2}$ and $\left[K_{1}, K_{2}\right]$; this last follows from the Jacobi identity [Eq. (3.9)]. The Killing tensors thus form both a subring and a Lie subalgebra of $S(M)[9,27]$ [a conventional, but useful, abuse of language: it is $\bar{S}(M)$, not $S(M)$ which has the structure of a Lie algebra].

The Hamiltonian $h: T^{*} M \rightarrow \mathbb{R}$ for the geodesics in $(M, g)$ is the function associated with the tensor $H=\frac{1}{2} G \in S^{2}(M)$, so an immediate corollary of Proposition 3.1 is that $K \in S(M)$ is a Killing tensor if, and only if, the associated function $k: T^{*} M \rightarrow \mathbb{R}$ is a constant of the motion for the geodesics in $M$.

In a general Riemannian manifold Killing's equation [Eq. (3.13)] has no nontrivial solutions. Equation (3.13") can be manipulated so as to express all the partial derivatives of the components of $K$ in terms of the partial derivatives of order $q$ or less together with the partial derivatives of the components of the Riemann tensor, $R$. These expressions, in turn, imply relations between the derivatives of the components of $R$ which are not usually satisfied [20,28]. The existence of solutions of non-zero valence is equivalent to the existence of non trivial constants of the motion which, when restricted to any fibre of $T^{*} M$, are analytic on the zero cross-section [20]. Any such function, $a$, can be expanded as a power series:

$$
a=a_{0}+a_{1}+a_{2}+\ldots
$$


where, for each $i, a_{i}: T^{*} M \rightarrow \mathbb{R}$ is the function associated with a tensor $A_{i} \in S^{i}(M)$. Clearly, if $a$ is a constant of the motion then each $A_{i}$ must be a Killing tensor [to see this, take the Poisson bracket of Eq. (3.14) with $h$ and, using Proposition 3.1, equate the terms of each degree in $\boldsymbol{p}$ to zero]. The existence of non-trivial constants of the motion of this form severely restricts the behaviour of the geodesic spray $X_{h}$ near its singularity on the zero cross-section of $T^{*} M$.

More general than a constant of the motion which is analytic (in $\boldsymbol{p}$ on the zero cross-section) is one that is rational (in $\boldsymbol{p})$ : a $\operatorname{Killing}$ pair $(A, B)$ is an ordered pair of tensors in $S(M)$ which satisfy the equation:

$A \cap[B, G]=B \cap[A, G]$.

If $A, B \in S(M)$ and if $a, b: T^{*} M \rightarrow \mathbb{R}$ are the corresponding functions on $T^{*} M$ then, by Proposition 3.1, $(A, B)$ is a Killing pair if, and only if, $a / b$ is a constant of the motion. The set of Killing pairs can thus be identified with a subalgebra of the algebra of constants of the motion (up to the abuse of language mentioned above); the Lie bracket is given by:

$$
\begin{aligned}
{[(A, B),(C, D)]=} & (B \cap D \cap[A, C]+A \cap C \cap[A, D]-A \cap D \cap[B, C] \\
& -B \cap C \cap[A, D], \quad B \cap B \cap D \cap D) .
\end{aligned}
$$

This subalgebra extends the algebra of Killing tensors: each Killing tensor $K$ can be identified with the Killing pair $(K, 1)$ where $1 \in S^{0}(M)$ is the unit constant function. Looked at in another way, the Killing pairs form a field under the obvious extensions of + and $\cap$. The second example below illustrates that this field can be strictly larger than the quotient field of the ring of Killing tensors.

Examples. 1) If $A, B \in S(M)$ are Killing tensors and $C \in S(M)$ is any other symmetric contravariant tensor then $(A \cap C, B \cap C)$ is a Killing pair: strictly speaking, this pair should be identified with $(A, B)$.

2) Let $M$ be a three dimensional manifold with local coordinates $(x, y, z)$ and let $l, m$, and $n$ be arbitrary functions of $z$. If the inverse metric $G$ has components:

$$
\left[\begin{array}{ccr}
x & \frac{1}{2}(x+y) & l(z) \\
\frac{1}{2}(x+y) & y & m(z) \\
l(z) & m(z) & n(z)
\end{array}\right]
$$

then the two vectors $A=\frac{\partial}{\partial x}$ and $B=\frac{\partial}{\partial y}$ form a Killing pair. In fact, $A$ and $B$ are projective Killing tensors ${ }^{2}$ of valence 1 (which is not to say that they are projective collineations) in that they satisfy the equations (in components):

$$
\begin{aligned}
& \nabla_{(\mathfrak{a}} A_{\mathfrak{b})}=A_{(\mathfrak{a}} X_{\mathfrak{b})} \\
& \nabla_{(\mathfrak{a}} B_{\mathfrak{b})}=B_{(\mathfrak{a}} X_{\mathfrak{b})}
\end{aligned}
$$

where $\nabla$ is the metric connexion and $X^{\mathfrak{a}}=\left(\frac{1}{2}, \frac{1}{2}, 0\right)$. If $\Gamma_{\mathfrak{b c}}^{\mathfrak{a}}$ is the metric Christoffel symbol, then $A_{\mathfrak{a}}$ and $B_{\mathfrak{a}}$ satisfy the covariant Killing equations:

$$
\tilde{\nabla}_{(\mathfrak{a}} A_{\mathfrak{b})}=\tilde{\nabla}_{(\mathfrak{a}} B_{\mathfrak{b})}=0
$$

\footnotetext{
${ }^{2}$ An unpublished concept due to A. Schild and P. Sommers.
} 
in the projectively related connexion with Christoffel symbol:

$$
\tilde{\Gamma}_{\mathrm{bc}}^{\mathrm{a}}=\Gamma_{\mathrm{bc}}^{\mathrm{a}}+\delta_{(\mathrm{b}}^{\mathrm{a}} X_{\mathrm{c})} \text {. }
$$

Except for special choices of $l, m$ and $n, X_{\mathfrak{b}}$ is not exact, $A$ and $B$ are not parallel to Killing vectors and $\tilde{\nabla}$ is not a metric connexion.

A second generalization extends the algebra of Killing tensors to include the conformal Killing tensors [31]: $C \in S^{p}(M)$ is a conformal Killing tensor if there exists $B \in S^{p-1}(M)$ such that:

$$
[C, G]=G \cap B .
$$

If $G$ is pseudo-Riemannian then $C \in S(M)$ is a conformal Killing tensor if and only if $c \in F\left(T^{*} M\right)$ is a constant of the motion for the null geodesics in $M$, for if $[c, h]=0$ on the zero energy surface, then $G$ must be a factor of $[C, G]$, that is $[C, G]=B \cap G$ for some $B$. The conformal Killing tensors also form both a subring and a Lie subalgebra of $S(M)$. This subalgebra may be further extended to include conformal Killing pairs, that is ordered pairs $(A, B), A \in S^{p}(M), B \in S^{q}(M)$, satisfying the equation:

$$
A \cap[B, G]-B \cap[A, G]=G \cap D
$$

for some $D \in S^{p+q-2}(M)$. Conformal Killing pairs define rational constants of the motion for null geodesics.

A Killing vector $X$ on $M$ (that is, a Killing tensor of valence 1) defines a symmetry in the sense that the associated local 1-parameter groups of diffeomorphisms are in fact groups of local isometries since:

$$
\mathscr{L}_{X} G=[X, G]=0 .
$$

In various generalizations of this notion, the associated local diffeomorphism groups are required to preserve weaker structures than the metric (for example, the projective or affine structures). Two particular cases will arise in $\S 4$ :

1) The vector field $X$ is a conformal Killing vector, that is the associated local diffeomorphism groups are groups of conformal isometries. A conformal Killing vector is characterized by the equation:

$$
\mathscr{L}_{X} G=u \cdot G ; \quad u \in F(M)
$$

and is, in fact (as the terminology suggests), the same thing as a conformal Killing tensor of valence 1 .

2) The vector field $X$ is rigid, that is it satisfies the equation:

$$
\mathscr{L}_{X} G=u \cdot X \cap X ; \quad u \in F(M) .
$$

If $\Delta: U \subset M \rightarrow M$ is a local diffeomorphism generated by $X$ then $\left(\Delta^{*}\right)^{-1}$ defines an isometry between the orthogonal complement of $X$ in $T_{m}^{*} M$ and the orthogonal complement of $X$ in $T_{\Delta(m)}^{*} M$, for each $m \in U$. Note that Eq. (3.21) is not quite the Born rigidity condition: if $X$ were a Born rigid motion then $\Delta_{*}$ would define an isometry between the orthogonal complement of $X$ in $T_{m} M$ and the orthogonal complement of $X$ in $T_{\Delta(m)} \mathrm{M}$ [23]. A conformally rigid vector field is characterized 
by the equation:

$$
\mathscr{L}_{X} G=u \cdot X \cap X+v \cdot G ; \quad u, v \in F(M) .
$$

The geometrical interpretation is similar.

A conformally rigid vector field can also be characterized by the linear dependence of $\mathscr{L}_{X} G, X \cap X$ and $G$ over $F(M)$. This observation suggests the following definitions: the degree of symmetry of a vector field $X$ is the smallest integer $s>0$ such that:

$$
\mathscr{L}_{X} G, \mathscr{L}_{X}^{2} G, \ldots \mathscr{L}_{X}^{s} G
$$

are linearly dependent over $F(M)$. The degree of symmetry of a Killing vector is 1 . The degree of conformal symmetry, the degree of rigidity and the degree of conformal rigidity are, respectively, the smallest positive integers $c, r$ and $k$ such that:

$$
\begin{aligned}
& G, \mathscr{L}_{X} G, \ldots \mathscr{L}_{X}^{c} G \\
& X \cap X, \mathscr{L}_{X} G, \ldots \mathscr{L}_{X}^{r} G
\end{aligned}
$$

and

$$
X \cap X, G, \mathscr{L}_{X} G, \ldots \mathscr{L}_{X}^{k} G
$$

are linearly dependent over $F(M)$ : these integers are finite for any vector field. A somewhat contrived geometrical interpretation can be assigned to, say, a vector field with symmetry degree $s$, but this is not particularly instructive; informally, $s$ measures how near a vector field is to being a Killing vector.

I end this section with some lemmas which will be needed later:

3.2. Proposition. Let $A, B \in S^{2}(M)$. If $\alpha \in \Lambda_{1}(M)$ and if $A(\alpha, \alpha)$ and $B(\alpha, \alpha)$ are constant on $M$ then:

$[A, B](\alpha, \alpha, \alpha)=-8 d \alpha(A(\alpha), B(\alpha))$.

Proof. First consider two vector fields $X, Y \in S^{1}(M)$ :

$$
\begin{aligned}
{[X \cap X, Y \cap Y](\alpha, \alpha, \alpha)=} & 4 \alpha(X) \cdot \alpha(Y) \cdot \alpha([X, Y]) \\
= & 4 \alpha(X) \cdot \alpha(Y) \cdot\{-2 d \alpha(X, Y)+X(\alpha(Y))-Y(\alpha(X))\} \\
= & -8 d \alpha(X \cap X(\alpha), Y \cap Y(\alpha)+2 X \cap X(\alpha, d(Y \cap Y(\alpha, \alpha))) \\
& -2 Y \cap Y(\alpha, d(X \cap X(\alpha, \alpha))) .
\end{aligned}
$$

$A$ and $B$ can be written:

$$
\left.\begin{array}{l}
A=\sum_{i} X_{i} \cap X_{i} \\
B=\sum_{j} Y_{j} \cap Y_{j}
\end{array}\right\} X_{i}, Y_{j} \in S^{1}(M)
$$

so the assertion follows from the linearity of the operations in Eq. (3.24) [using $d(A(\alpha, \alpha))=0=d(B(\alpha, \alpha))]$.

3.3. Proposition. Let $A, B \in S^{2}(M)$. If $\alpha \in \Lambda_{1}(M)$ is closed then:

$3[A, B](\alpha)=[A(\alpha), B]+[A, B(\alpha)] \in S^{2}(M)$. 
Proof. Again, first consider two vector fields $X, Y \in S^{1}(M)$ :

$$
\begin{aligned}
3[X \cap X, Y \cap Y](\alpha) & =12(X \cap[X, Y] \cap Y)(\alpha) \\
& =4(\alpha(X) \cdot Y \cap[X, Y]+\alpha([X, Y]) \cdot X \cap Y+\alpha(Y) \cdot X \cap[X, Y]) \\
& =[X \cap X(\alpha), Y \cap Y]+[X \cap X, Y \cap Y(\alpha)]+4 d \alpha(X, Y) .
\end{aligned}
$$

As in Proposition 3.2, the assertion now follows by linearity (and the closure of $\alpha$ ).

An eigenform of a tensor $A \in S^{2}(M)$ is a 1-form $\alpha$ such that the vectors $A(\alpha)$ and $G(\alpha)$ are parallel at each point of $M$. If $M$ is $n$-dimensional then each tensor in $S^{2}(M)$ has at most $n$ independent eigenforms.

3.4. Proposition. If $\alpha$ is a closed eigenform of the Killing tensor $K \in S^{2}(M)$ and if $T \in S^{1}(M)$ is the vector field defined by:

$$
\left.\begin{array}{rl}
\begin{array}{r}
u \\
\cdot T
\end{array} & =K(\alpha) \\
w \cdot T & =G(\alpha)
\end{array}\right\} u, w \in F(M)
$$

then:

1) $\mathscr{L}_{T}(w / u \cdot K-G)=0$

2) $T(u / w)=0$.

Proof. First note that, since $\alpha(T)=1, \mathscr{L}_{T} \alpha=0$. The tensors $K$ and $G$ may be decomposed:

$$
\left.\begin{array}{rl}
K & =\bar{K}+u \cdot T \cap T \\
G & =\bar{G}+w \cdot T \cap T
\end{array}\right\} \bar{K}, \bar{G} \in S^{2}(M)
$$

where:

$$
\begin{aligned}
\bar{K}(\alpha) & =0=\bar{G}(\alpha), \\
{[\bar{G}, u](\alpha) } & =0=[\bar{K}, w](\alpha), \\
{[\bar{K}, \bar{G}](\alpha) } & =0 \quad(\text { by Proposition 3.3), } \\
{[T, \bar{G}](\alpha) } & =\mathscr{L}_{T} \bar{G}(\alpha)=0=[T, \bar{K}](\alpha) .
\end{aligned}
$$

Expanding:

$$
0=3[K, G](\alpha)=3[\bar{K}+u \cdot T \cap T, \bar{G}+w \cdot T \cap T](\alpha)
$$

and collecting the non-zero terms:

$$
\begin{aligned}
0= & 2 u \cdot[T, \bar{G}]+2 w \cdot[\bar{K}, T]+2([u, \bar{G}]+[\bar{K}, w]) \cap T+6(u \cdot T(w) \\
& -w \cdot T(u)) T \cap T .
\end{aligned}
$$

Contracting Eq. (3.33) with $\alpha$ gives:

$$
\begin{aligned}
u \cdot T(v)-w \cdot T(u) & =0, \\
u \cdot \mathscr{L}_{T} \bar{G}-w \cdot \mathscr{L}_{T} \bar{K} & =0,
\end{aligned}
$$


whence:

$$
\mathscr{L}_{T}(\bar{G}-w / u \cdot \bar{K})=\mathscr{L}_{T}(G-w / u \cdot K)=0 .
$$

\section{§ 4. Separable Systems}

This section deals with local questions: all closed forms are exact and each solution of the Hamilton-Jacobi equation is defined over the whole of the base manifold.

Let $(M, g)$ be a Riemannian or Lorentzian manifold (that is $g$ is either positive definite or it has signature,,,,$+--- \ldots)$; let $h: T^{*} M \rightarrow \mathbb{R}$ be the Hamiltonian for the geodesics in $M$. The associated Hamilton-Jacobi equation has a large number of solutions: any suitably parameterized hypersurface orthogonal congruence of geodesics defines a particular solution and any $n$-parameter family of such congruences defines a complete solution. In the following, I shall restrict my attention to solutions for which $h \geqq 0$; the only place where this plays an important role is in the lemma needed to prove Proposition 4.1.

Let $\alpha \in \wedge_{1}(M)$ be a closed 1-form and let $T \in S^{1}(M)$ be a vector field normalized so that $\alpha(T)=1$. If $X \in S^{1}(M)$ and $\varphi \in \wedge_{1}(M)$ then the projections $\perp X$ and $\perp \varphi$ relative to $(T, \alpha)$ are defined by:

$$
\left.\begin{array}{l}
\perp X=X-\alpha(X) \cdot T \\
\perp \varphi=\varphi-\varphi(T) \cdot \alpha
\end{array}\right\} .
$$

Note that $\alpha(\perp X)=0=(\perp \varphi)(T)$, that $\varphi(\perp X)=(\perp \varphi)(X)$, that $\perp$ extends naturally to tensor fields and that it commutes with $\mathscr{L}_{T}$.

A closed solution $\varphi \in \Lambda_{1}(M)$ of the Hamilton-Jacobi equation is said to separate with respect to $(T, \alpha)$ if:

$$
\mathscr{L}_{T}(\perp \varphi)=0 .
$$

If local coordinates $\left\{x^{\alpha}\right\}$ are chosen so that $T=\frac{\partial}{\partial x^{1}}$ and $\alpha=d x^{1}$ then $\varphi$ may be written:

$$
\varphi_{\mathrm{a}}=\partial_{\mathrm{a}} s ; \quad s \in F(M)
$$

and the separability condition assumes its more familiar form:

$$
s\left(x^{\mathfrak{a}}\right)=s_{1}\left(x^{1}\right)+s_{2}\left(x^{2}, x^{3} \ldots x^{n}\right) .
$$

The separation is said to be trivial if:

$$
\mathscr{L}_{T} \varphi=0 .
$$

Equation (4.5) implies. Eq. (4.2) since $\mathscr{L}_{T}$ and $\perp$ commute.

The pair $(T, \alpha)(\alpha(T)=1, d \alpha=0, G(\alpha, \alpha) \neq 0)$ will be called a separable system if there exists a complete solution $\Psi: M \times V \subset M \times \mathbb{R}^{n} \rightarrow T^{*} M$ such that $\psi_{v}$ separates with respect to $(T, \alpha)$ for each $v \in V$ (in the Lorentz case, each $\psi_{v}$ must also be non-spacelike); $(T, \alpha)$ is a trivial separable system if the separation is trivial for each $v$. It is an orthogonal separable system if $T$ is parallel to $G(\alpha)$. Two separable 
systems are compatible if there exists a complete solution which separates with respect to both systems. In the Lorentz case, $(T, \alpha)$ is said to be a separable system for the null energy surface if there exists a complete null solution $\Psi: M \times V \subset M \times$ $\mathbb{R}^{n-1} \rightarrow N \subset T^{*} M$ such that $\psi_{v}$ separates with respect to $(T, \alpha)$ for each $v(N$ is the null energy surface). By an obvious extension, one can also talk about compatible separable systems for the null energy surface and so on.

It is immediately clear that if $(T, \alpha)$ is a trivial separable system then $T$ must be a Killing vector. To see this, choose a complete solution $\Psi: M \times V \subset M \times \mathbb{R}^{n} \rightarrow$ $T^{*} M$ which separates trivially with respect to $(T, \alpha)$, so that:

$$
\mathscr{L}_{T} \Psi_{v}=0 ; \quad v \in V .
$$

For each $v, G\left(\psi_{v}, \psi_{v}\right)$ is constant, hence:

$$
0=\mathscr{L}_{T} G\left(\psi_{v}, \psi_{v}\right)=\left(\mathscr{L}_{T} G\right)\left(\psi_{v}, \psi_{v}\right) ; \quad v \in V
$$

As $(m, v) \in M \times V$ varies, $\Psi(m, v)$ fills out an open set in $T^{*} M$, so it follows from Eq. (4.7) that $\mathscr{L}_{T} G=0$. If $(T, \alpha)$ is only a separable system for the null energy surface then $T$ need only be a conformal Killing vector (this can be established by a similar argument).

Note that if $(T, \alpha)$ is a trivial separable system then so is $(T, \beta)$ for any closed 1 -form $\beta$ such that $\beta(T)=1$, so it makes sense to refer to $T$ alone as a trivial separable system.

One final definition: $T^{*} M^{+}$denotes the subset of $T^{*} M$ on which $h>0$.

The first aim of this section is to establish that essentially all separable systems are either trivial or orthogonal. A few preliminary lemmas are needed:

4.1. Proposition. If $X$ is a Killing vector then:

1) $X$ is a trivial separable system.

2) If $(T, \alpha)$ is any separable system such that $[X, T]=0$ and $\alpha(X)=0$ then $(T, \alpha)$ and $X$ are compatible.

Proof. Let $\Psi: M \times V \subset M \times \mathbb{R}^{n} \rightarrow T^{*} M^{+}$be a (local) complete solution. Restricting, if necessary, the domain of definition $\Psi$, one may distinguish two possible cases:

a) $X\left(\psi_{v}(X)\right) \neq 0 ; \quad v \in V$,

b) $X\left(\psi_{v}(X)\right)=0 ; \quad v \in V$.

In either case, it is possible to construct from $\Psi$ a new complete solution $\Phi$ which separates trivially with respect to $X$. Further, if the original solution $\Psi$ separates with respect to $(T, \alpha)$ then so does this new solution $\Phi$. The following lemma establishes that if Eq. (4.9) holds then $\psi_{v}(X)$ must be constant for each $v$.

Lemma. Let $\psi \in \wedge_{1}(M)$ be a closed solution of the Hamilton-Jacobi equation (timelike or null in the Lorentz case). If $X$ is a Killing vector and $y=\psi(X)$ then either $X(y) \neq 0$ or $y$ is constant.

Proof. Suppose that $X(y)=0$. Since $\psi$ is closed:

$d y=d(\psi(X))=\mathscr{L}_{X} \psi$. 
But $G(\psi, \psi)$ is constant, so:

$$
0=\mathscr{L}_{X}(G(\psi, \psi))=2 G\left(\psi, \mathscr{L}_{X} \psi\right)=2 G(\psi, d y) .
$$

Operating again with $\mathscr{L}_{X}$ gives $G(d y, d y)=0$, since $\mathscr{L}_{X} d y=d(X(y))=0$. If $G$ is Riemannian then $d y=0$ and $y$ is constant. If $G$ is Lorentzian then $d y$ is null and orthogonal to $\psi$. But $\psi$ is non-spacelike; $\psi$ must, therefore, be parallel to $d y$, giving $\psi(X)=0$ that is $y=0$.

It follows that, whichever of Eqs. (4.8) and (4.9) holds, it is possible to find, for each $v \in V$, a hypersurface $S_{v} \subset M$ which is transversal to $X$ and on which $\psi_{v}(X)$ is constant (this may not be true globally). Each $S_{v}$ may be chosen so as to pass through some fixed point $m_{0} \in M$. Further, if $T$ and $\alpha$ are as in the second part of the proposition and if $\Psi$ separates with respect to $(T, \alpha)$ then each $S_{v}$ may also be assumed to be tangent to $T$, since:

$$
\begin{aligned}
T\left(\psi_{v}(X)\right. & =T\left(\left(\perp \psi_{v}\right)(X)\right) \\
& =\left(\perp \psi_{v}\right)\left(\mathscr{L}_{T} X\right)+\left(\mathscr{L}_{T}\left(\perp \psi_{v}\right)\right)(X)=0 .
\end{aligned}
$$

Here $\perp$ is the projection relative to $(T, \alpha)$. Now, for each $v \in V$, define the 1 -form $\varphi$, by:

$$
\begin{aligned}
& \varphi_{v}=\psi_{v} \text { on } S_{v}, \\
& \mathscr{L}_{X} \varphi_{v}=0 .
\end{aligned}
$$

This is a good definition (at least near $S_{v}$ ) since $X$ is transversal to $S_{v}$. Since $\mathscr{L}_{X} G=0$, $G\left(\varphi_{v}, \varphi_{v}\right)=G\left(\psi_{v}, \psi_{v}\right)$ is constant. To see that $\varphi_{v}$ is also closed, choose any two vector fields $Y, Z \in S^{1}(M)$ which are tangent to $S_{v}$ and which satisfy:

$$
[X, Y]=[X, Z]=[Y, Z]=0 \text {. }
$$

Computing:

$$
\begin{aligned}
-2 d \varphi_{v}(X, Y) & =-Y\left(\varphi_{v}(X)\right)+X\left(\varphi_{v}(Y)\right) \\
& =\mathscr{L}_{X}\left(\varphi_{v}(Y)\right) \\
& =\varphi_{v}\left(\mathscr{L}_{X} Y\right)+\left(\mathscr{L}_{X} \varphi_{v}\right)(Y) \\
& =0
\end{aligned}
$$

and, on $S_{v}$ :

$$
\begin{aligned}
2 d \varphi_{v}(Y, Z) & =Y\left(\varphi_{v}(Z)\right)-Z\left(\varphi_{v}(Y)\right) \\
& =Y\left(\psi_{v}(Z)\right)-Z\left(\psi_{v}(Y)\right) \\
& =2 d \psi_{v}(Y, Z)=0 .
\end{aligned}
$$

Thus $d \varphi_{v}=0$ on $S_{v}$; but $\mathscr{L}_{X} d \varphi_{v}=d \mathscr{L}_{X} \varphi_{v}=0$ so $d \varphi_{v}=0$ everywhere.

Hence $\Phi: M \times V \subset M \times \mathbb{R}^{n} \rightarrow T^{*} M: \Phi:(m, v) \mapsto\left(m, \varphi_{v(m)}\right)$ is a complete solution of the Hamilton-Jacobi equation which separates trivially with respect to $X$ (the completeness follows from the fact that $\left.\left.\Phi\right|_{m_{0} \times V}=\left.\Psi\right|_{m_{0} \times V}\right)$.

Suppose, now, that the original solution $\Psi$ was chosen so as to separate with respect to $(T, \alpha)$. All that remains to complete the proof is to show that each $\varphi_{v}$ 
also separates with respect to $(T, \alpha)$ : For each $v \in V, T$ is tangent to $S_{v}$ and so, on $S_{v}$ :

$$
\mathscr{L}_{T}\left(\perp \varphi_{v}\right)=\mathscr{L}_{T}\left(\perp \psi_{v}\right)=0 .
$$

Also:

$$
\mathscr{L}_{X}\left(\mathscr{L}_{T}\left(\perp \varphi_{v}\right)\right)=\mathscr{L}_{T}\left(\mathscr{L}_{X}\left(\perp \varphi_{v}\right)\right)=\mathscr{L}_{T}\left(\perp \mathscr{L}_{X} \varphi_{v}\right)=0
$$

so that $\mathscr{L}_{T}\left(\perp \varphi_{v}\right)=0$ everywhere.

Proposition 4.1 has three corollaries; the first is the standard result:

1. Corollary. If $\left\{X_{i} ; i=1,2 \ldots\right\}$ is a commuting set of Killing vectors then there exists a complete solution $\Phi: M \times V \subset M \times \mathbb{R}^{n} \rightarrow T^{*} M$ which separates trivially with respect to each $X_{i}$.

2. Corollary. Let $(T, \alpha)$ be a separable system and let $X$ be a Killing vector such that $\alpha(X)=0$ and $[X, T]=0$. If $b: M \rightarrow \mathbb{R}$ is any function such that $d b$ is parallel to $\alpha$ then $(T+b \cdot X, \alpha)$ is a separable system.

Proof. Let $\perp$ be the projection relative to $(T, \alpha)$ and let $\perp^{\prime}$ be the projection relative to $(T+b \cdot X, \alpha)$; thus:

$$
\begin{aligned}
& \perp^{\prime} \beta=\perp \beta-b \cdot \beta(X) \cdot \alpha=\beta-\beta(T+b \cdot X) \cdot \alpha ; \quad \beta \in \wedge_{1}(M), \\
& \beta(X)=(\perp \beta)(X)=\left(\perp^{\prime} \beta\right)(X) ; \quad \beta \in \wedge_{1}(M) .
\end{aligned}
$$

Let $\Phi: M \times V \subset M \times \mathbb{R}^{n} \rightarrow T^{*} M$ be a complete solution which separates with respect to $(T, \alpha)$ and also, trivially, with respect to $X$. A direct calculation gives:

$$
\begin{aligned}
\mathscr{L}_{T+b . X}\left(\perp^{\prime} \varphi_{v}\right)= & \mathscr{L}_{T}\left(\perp \varphi_{v}-b \cdot\left(\perp \varphi_{v}\right)(X) \cdot \alpha\right)+b \cdot \mathscr{L}_{T}\left(\varphi_{v}-\varphi_{v}(T+b \cdot X) \cdot \alpha\right) \\
& +\left(\varphi_{v}-\varphi_{v}(T+b \cdot X) \cdot \alpha\right)(X) \cdot d b ; \quad v \in V \\
= & -\varphi_{v}(X) \cdot T(b) \cdot \alpha+\varphi_{v}(X) \cdot d b \\
= & 0 . \quad \square
\end{aligned}
$$

3. Corollary. Let $\left\{X_{i} ; i=1,2 \ldots\right\}$ be a commuting set of Killing vectors. If the functions $b_{i}: M \rightarrow \mathbb{R}$ and the 1 -form $\alpha$ satisfy:

1) $d b_{i}$ is parallel to $\alpha$ for each $i$,

2) $\alpha(T)=1 ; T=b_{1} \cdot X_{1}+b_{2} \cdot X_{2}+\ldots$,

3) $d \alpha=0$,

then $(T, \alpha)$ is a separable system.

Proof. Let $\Phi: M \times V \subset M \times \mathbb{R}^{n} \rightarrow T^{*} M$ be a complete solution which separates trivially with respect to each $X_{i}$ and let $\perp$ be the projection relative to $(T, \alpha)$. A direct calculation gives:

$$
\begin{aligned}
\mathscr{L}_{T}\left(\perp \varphi_{v}\right) & =\mathscr{L}_{T}\left(\varphi_{v}\right)-\left(\mathscr{L}_{T}\left(\varphi_{v}\right)\right)(T) \cdot \alpha ; \quad v \in V \\
& =\sum_{i}\left\{\varphi_{v}\left(X_{i}\right) \cdot d b_{i}-\left(\varphi_{v}\left(X_{i}\right) \cdot d b_{i}\right)(T) \cdot \alpha\right\} \\
& =0
\end{aligned}
$$

since $d b_{i}=T\left(b_{i}\right) \cdot \alpha$ for each $i$.

The direction of the argument can now be defined more precisely: it will be shown in the following that the only possible separable systems are those which 
can be obtained from orthogonal separable systems by transformations of the type arising in Corollary 2 and those which can be constructed from abelian Lie algebras of Killing vectors as in Corollary 3.

Let $(T, \alpha)$ be a separable system, let $\Phi: M \times V \subset M \times \mathbb{R}^{n} \rightarrow T^{*} M$ be a complete solution which separates with respect to $(T, \alpha)$ and let $S$ be an integral surface of $\alpha$ ( $S$ certainly exists locally since $\alpha$ is closed). Define $\bar{k}: V \rightarrow \mathbb{R}$ by:

$$
\bar{k}(v)=\left.\varphi_{v}(T)\right|_{S} ; \quad v \in V .
$$

$\left[\varphi_{v}(T)\right.$ is constant on $S$ for each $v \in V$ by separability and the closure of $\left.\varphi_{v}.\right]$ Put:

$$
k=\bar{k} \circ \Pi_{2} \circ \Phi^{-1}: T^{*} M \rightarrow \mathbb{R}
$$

where $\Pi_{2}: M \times V \rightarrow V$ is the projection map. By Theorem 2.1, $k$ is a (local) constant of the motion. An explicit form for $k$ may be found as follows: define the function $u \in S^{0}(M)$ and the tensor fields $C \in S^{2}(M), D \in S^{1}(M)$ by:

$$
\begin{array}{ll}
u=G(\alpha, \alpha) \quad \text { on } \quad S ; \quad T(u)=0, \\
C=\perp G \quad \text { on } \quad S ; \quad \mathscr{L}_{T} C=0, \\
D=\perp G(\alpha) \quad \text { on } \quad S ; \quad \mathscr{L}_{T} D=0 .
\end{array}
$$

[Here $\perp$ is the projection relative to $(T, \alpha)$.] These are good definitions, at least locally, since $T$ is transversal to $S$. At points on $S$, the Hamilton-Jacobi equation takes the form:

$$
\left.\left(u \cdot \bar{k}^{2}(v)+2 \bar{k}(v) \cdot D\left(\perp \varphi_{v}\right)+C\left(\perp \varphi_{v}, \perp \varphi_{v}\right)\right)\right|_{S}=2 E_{v}
$$

where $E_{v} \in \mathbb{R}$ is the energy of $\varphi_{v}$ [that is, the value of $h$ on the surface $\left.\Phi_{v}(M) \subset T^{*} M\right]$. For fixed $v \in V$, every quantity in Eq. (4.29) is Lie propagated by $T$, so the equation must be true without the restriction to $S$. Substituting:

$$
G\left(\varphi_{v}, \varphi_{v}\right)=2 E_{v}
$$

gives:

$$
\bar{k}^{2}(v)+\bar{k}(v) \cdot B\left(\varphi_{v}\right)+A\left(\varphi_{v}, \varphi_{v}\right)=0
$$

where ${ }^{3}$ :

$$
\begin{aligned}
& A=\frac{1}{u} \cdot(C-G) \in S^{2}(M), \\
& B=\frac{2}{u} \cdot D \in S^{1}(M) .
\end{aligned}
$$

Allowing $v$ to vary over $V$, deduce that $k$ is a root of the quadratic equation:

$$
k^{2}+k \cdot b+a=0
$$

where $a, b: T^{*} M \rightarrow \mathbb{R}$ are the functions on $T^{*} M$ defined by $A$ and $B$. Taking the Poisson bracket of Eq. (4.34) with the Hamiltonian $h$ :

$k \cdot[b, h]+[a, h]=0$.

\footnotetext{
${ }^{3}$ It is assumed that $\alpha$ is not null.
} 
There are now three possibilities:

1) $[b, h]=[a, h]=0$ : in this case, both roots of Eq. (4.34) are constants of the motion, $B$ is a Killing vector and $A$ is a Killing tensor (Proposition 3.1). In particular, this case arises whenever $(T, \alpha)$ is an orthogonal system $(b=0)$.

2) Only one root of Eq. (4.34) is a constant of the motion and ( $[A, G],[B, G])$ is a Killing pair, but it is trivial in the sense that:

$$
[A, G]=-[B, G] \cap X
$$

for some $X \in S^{1}(M)$. In this case, $X$ is a Killing vector and $k=x$. In particular, this case arises whenever $(T, \alpha)$ is a trivial separable system (see the example below).

3) $([A, G],[B, G])$ is a non-trivial Killing pair.

Case 3 cannot occur. One way to see this is to eliminate $k$ between Eqs. (4.34) and (4.35). This yields the tensor identity:

$$
[A, G] \cap[A, G]-[A, G] \cap[B, G] \cap B+[B, G] \cap[B, G] \cap A=0 .
$$

Call $U \in S(M)$ a factor of $V \in S(M)$ whenever there exists $W \in S(M)$ such that $V=U \cap W$ and note that the unique factorization theorem holds in the polynomial ring $\bar{S}(M)$. Rearranging Eq. (4.37):

$$
[A, G] \cap\left[A-\frac{1}{2} B \cap B, G\right]=-[B, G] \cap[B, G] \cap A .
$$

Any common factor of $[B, G]$ and $\left[A-\frac{1}{2} B \cap B, G\right]$ must also be a factor of $[A, G]$. It follows that either $[B, G]$ is a factor of $[A, G]$ or $[B, G]=0$.

Example (Separation of an ignorable coordinate). Suppose that $X$ is a Killing vector and that $\alpha$ is any closed 1 -form such that $\alpha(X)=1$. By Proposition 4.1 $(X, \alpha)$ is a separable system. If $Y=\frac{1}{u} \cdot G(\alpha) \in S^{1}(M)$ then, whatever the choice of $S$ :

$$
\begin{aligned}
& B=2 Y-2 X ; \quad[B, G]=2[Y, G], \\
& A=-X \cap(2 Y-X) ; \quad[A, G]=-2 X \cap[Y, G] .
\end{aligned}
$$

In this case, the two roots of Eq. (4.34) are $k_{1}=x$ and $k_{2}=x-2 y$. For a general choice of $\alpha$, only $k_{1}$ is a constant of the motion. It is clear from Eqs. (4.39) and (4.40) that $[B, G]$ is a factor of $[A, G]$ and thus that this example is covered by Case 2.

Historical Note. A separable coordinate system $\left\{x^{a}\right\}$ on $M$ is characterized by the fact that $\left(\frac{\partial}{\partial x^{a}}, d x^{a}\right)$ is a compatible separable system for each $a$; such coordinate systems were studied extensively during the late nineteenth and early twentieth centuries first by Liouville [18] and later by Stäckel [28], Levi-Civita [17], Dall'Acqua [6], Burgatti [4], and others. Their most powerful tool was the integrability condition first formulated by Levi-Civita and explained by him in a letter to Stäckel (1904) [17]. Stäckel knew that orthogonal separability gives rise to quadratic constants of the motion and it is implicit in the work of Dall'Acqua (1912) [6] that, for a general separable coordinate system, the constants of the motion are made up of Killing vectors and valence two Killing tensors. It is 
impossible to be certain as to how much of the following was known to these researchers, though Theorem 4.1 would have come as no surprise to anyone who had spent any time working out examples of separable coordinate systems from Levi-Civita's integrability condition.

The method developed here is more general than Levi-Civita's: his integrability condition depends in an essential way on the assumption that each of the coordinates is separable. Cases 1 and 2 above correspond to what Levi-Civita calls indices of the first and second kind.

In the construction described above for the constant of the motion $k: T^{*} M \rightarrow \mathbb{R}$ a particular integral surface $S$ of $\alpha$ was chosen arbitrarily as an initial surface. In general, a different choice $S^{\prime}$ will give rise to a different constant of the motion $k^{\prime}$. A maximum of $n$ (the dimension of $M$ ) vertically independent constants of the motion can be constructed in this way; these will commute with each other (Theorem 2.1). I continue with a separate examination of the two possible cases:

Case (1). For each choice of $S, A$ is a Killing tensor and $B$ is a Killing vector.

Call one particular integral surface of $\alpha S_{0}$ and measure the parameter $t$ on the integral curves of $T$ from $S_{0}$ (thus $d t=\alpha$, locally). Each integral surface $S_{t}$ of $\alpha$, and each of the quantities $A_{t}, B_{t}$ etc. defined as in Eqs. (4.26)-(4.33) by taking $S_{t}$ as initial surface in place of $S$, will be labelled by the appropriate value of $t$.

It is clear from Eqs. (4.28) and (4.33) that, if $P_{t}$ is the orthogonal projection of $T$ into $S_{t}$, then:

1) On $S_{t}: B_{t}=-2 P_{t} ; t \in \mathbb{R}$.

2) $\left[T, B_{t}\right]=0 ; \quad t \in \mathbb{R}$.

3) $\alpha\left(B_{t}\right)=0 ; t \in \mathbb{R}$.

Also, operating on the defining relation:

$$
B_{s}=\frac{2}{u_{s}} \cdot D_{s}
$$

with $\mathscr{L}_{B_{t}}$ gives:

$$
\left[B_{t}, B_{s}\right]=0 ; \quad s, t \in \mathbb{R}
$$

since $B_{t}\left(u_{s}\right)=0$ and $\left[B_{t}, D_{s}\right]=0$ [using Eqs. (4.42) and (4.43) together with the fact that $B_{t}$ is a Killing vector]. Now, only a finite number of the $B_{t}$ 's can be linearly independent over $\mathbb{R}$ so each $B_{t}$ must be a linear combination (with coefficients in $\mathbb{R}$ ) of some basis set of commuting Killing vectors $\left\{B_{1}, B_{2} \ldots B_{r}\right\}$, say. Thus, using Eq. (4.41),

$$
T=b_{1} \cdot B_{1}+b_{2} \cdot B_{2}+\ldots+b_{r} \cdot B_{r}+\bar{T}
$$

where $\bar{T}$ is a vector field orthogonal to each $S_{t}$ and the functions $b_{i}: M \rightarrow \mathbb{R}$ can be expressed as functions of $t$ alone. Application $r$ times of the second corollary to Proposition 4.1 reduces $(T, \alpha)$ to the orthogonal separable system $(\bar{T}, \alpha)$. The first case, therefore, is essentially the orthogonal case.

How many constants of the motion can be constructed from a separable system of this type? Consider, first, the case where $(T, \alpha)$ itself is orthogonal; then 
$B_{t}=0$ and each tensor $C_{t}$ is constructed by projecting the contravariant metric $G$ into $S_{t}$ and Lie propagating along $T$ ( $\perp$ is now simply the orthogonal projection relative to $T$ ). Suppose that it is possible to find a maximum of $q$ values of $t$ such that the corresponding Killing tensors $A_{t}$ and the metric $G$ are linearly independent over $F(M)$. By a straightforward exercise in analysis, it is possible to check that $q-1$ is precisely the maximum integer $m$ such that:

$$
\mathscr{L}_{T}^{m}(\perp G), \mathscr{L}_{T}^{m-1}(\perp G) \ldots \mathscr{L}_{T}(\perp G), G \text { and } T \cap T
$$

are linearly independent over $F(M)$, that is that $q$ is precisely the conformal rigidity of $T$. Thus:

4.2. Proposition. If $(T, \alpha)$ is an orthogonal separable system and if the degree of conformal rigidity of $T$ is $q$ then $\left\{A_{t}, G ; t \in \mathbb{R}\right\}$ generate a submodule of the $F(M)$ module $S^{2}(M)$ of rank $q+1$; each element of this submodule admits $\alpha$ as a closed eigenform.

The last part of the proposition follows from Eqs. (4.32) and (4.27).

Example. If $\alpha$ is a closed 1-form such that $T=G(\alpha) / G(\alpha, \alpha)$ is a Killing vector then, by Proposition 4.1, $(T, \alpha)$ is an orthogonal separable system. Regardless of the choice of $S, A=T \cap T$. The conformal rigidity of $T$ is 1 [since $\mathscr{L}_{T} G=0$ and $0, G$ and $T \cap T$ are trivially linearly dependent over $F(M)] ; A$ and $G$ span a rank 2 submodule of $S^{2}(M)$.

Roughly speaking, Proposition 4.2 states that the less symmetry $(M, g)$ has with respect to $T$, the more constants of the motion can be constructed from $(T, \alpha)$.

When $(T, \alpha)$ is not orthogonal, the rank of the submodule generated by $\left\{A_{t}, G ; t \in \mathbb{R}\right\}$ can exceed the conformal rigidity of $T$ by $\frac{1}{2} r(r+1)$ : this submodule contains, in addition to the Killing tensors constructed from the orthogonal system $(\bar{T}, \alpha)$, the linear span of $\left\{B_{i} \cap B_{j} ; i, j=1,2 \ldots r\right\}$.

Remark. Though the number of vertically independent constants of the motion which can be constructed from a single complete solution of the HamiltonJacobi equation cannot exceed $n$ (the dimension of $M$ ) it is possible that more than $n$ linearly independent [over $F(M)$ ] Killing tensors can be constructed from a single orthogonal separable system (an example is provided by the Kerr metric in general relativity, see $\S 6$ ). However it is the number of vertically independent constants rather than the number of linearly independent Killing tensors which is of practical interest. This number is also related to the conformal rigidity, but in a more complicated way. To give an example: suppose that $n=5$ and that only two vertically independent constants of the motion can be constructed from the orthogonal separable system $(T, \alpha)$. If $A, B, C \in S^{2}(M)$ are Killing tensors and if the corresponding functions $a, b, c \in F\left(T^{*} M\right)$ are vertically dependent then, in local coordinates:

$$
A_{(\mathrm{i}}^{[\mathrm{a}} B_{\mathrm{i}}^{\mathrm{b}} C_{\mathrm{f})}^{\mathrm{c}]}=0 .
$$

Denote by $\mathscr{T}$ the submodule of $S^{2}(M)$ generated by $G$ and the Killing tensors constructed from $(T, \alpha)$. If Eq. (4.46) is satisfied for each $A, B, C \in \mathscr{T}$ then, for certain, the rank of $\mathscr{T}$ cannot exceed 3 and so the conformal rigidity of $T$ cannot exceed 2. 
One other remark is worth stating as a separate proposition:

4.3. Proposition. If $(M, g)$ admits no Killing vectors then all separable systems in $M$ are orthogonal.

Turning now to the second case:

Case (2). For each choice of $S,[B, G] \neq 0$ is a factor of $[A, G]$.

Using the integral surface $S_{t}$ of $\alpha$ as initial surface in place of $S$ the construction yields a Killing vector $X_{t}$ given by (in the same notation as case 1):

$$
\left[B_{t}, G\right] \cap X_{t}+\left[A_{t}, G\right]=0 ; \quad t \in \mathbb{R} .
$$

It follows from Eq. (4.24) that $T=X_{t}$ on $S_{t}$ and from Theorem 2.1 that $\left[X_{t}, X_{s}\right]=0$ for each $s, t \in \mathbb{R}$. Again, not more than a finite number ( $r$, say) of the $X_{t}$ 's can be linearly independent over $\mathbb{R}$, so each must be a linear combination (with coefficients in $\mathbb{R}$ ) of some commuting basis set $\left\{X_{1}, X_{2} \ldots X_{r}\right\}$ of Killing vectors. Hence:

$$
T=b_{1} \cdot X_{1}+b_{2} \cdot X_{2}+\ldots+b_{r} \cdot X_{r}
$$

where each $b_{i}: M \rightarrow \mathbb{R}$ can be expressed as a function of $t$ alone: the separable system $(T, \alpha)$ is of precisely the type described in the third corollary to Proposition 4.1. This second case is, then, essentially the trivial case.

The number of vertically independent constants of the motion which can be constructed from a separable system of this type is equal to the dimension of the abelian Lie algebra generated by $\left\{X_{1}, X_{2} \ldots X_{r}\right\}$ since a set of commuting Killing vectors is linearly independent [either over $\mathbb{R}$ or over $F(M)$ ] if and only if the corresponding constants of the motion are independent.

A separable system of the type described in the third corollary to Proposition 4.1 will be called a type II separable system; a type I separable system is one which can be obtained from an orthogonal system by a finite number of transformations of the type described in the second corollary to Proposition 4.1. Collecting together the above results:

Theorem 4.1. All separable systems in $(M, g)$ are either of type I or of type $I I$.

Proposition 4.1 provides necessary and sufficient conditions for there to exist type II separable systems, namely the existence of an abelian isometry group of the appropriate dimension (at least locally). It is also of interest to inquire whether there exist sufficient conditions for the existence of type I separable systems. The following generalizes a theorem due to Eisenhart [7]: it is the converse of Proposition 4.2 in the maximal case where $n$ vertically independent constants of the motion are generated by an orthogonal separable system:

4.2. Theorem. If $M$ is $n$ dimensional and if $(M, \boldsymbol{g})$ admits $n-1$ valence-2 Killing tensors $K_{1}, K_{2} \ldots K_{n-1}$ such that:

1) $k_{1}, k_{2} \ldots k_{n-1}, h: T^{*} M \rightarrow \mathbb{R}$ are vertically independent in some neighbourhood $U \subset T^{*} M^{+}$.

2) $\left[K_{i}, K_{j}\right]=0 ; i, j=1,2 \ldots n-1$.

3) $K_{1}, K_{2} \ldots K_{n-1}$ have a common closed eigenform $\alpha \in \Lambda_{1}(M)$ then $(T, \alpha)$ is an orthogonal separable system, where $T=G(\alpha) / G(\alpha, \alpha)$. 
Proof. Write $K_{n}=G$. Since the functions $k_{1}, k_{2} \ldots k_{n} \in F\left(T^{*} M\right)$ are vertically independent in $U$, it is possible to define a local trivialization $\Phi: M \times V \subset M \times \mathbb{R}^{n} \rightarrow$ $U \subset T^{*} M^{+}$by:

$$
\left(k_{i} \circ \Phi\right)(m, v)=v_{i} ; \quad v=\left(v_{1}, v_{2} \ldots v_{n}\right) \in V, \quad m \in M
$$

( $M$ may have to be restricted here). By Proposition 3.2:

$$
\begin{aligned}
0 & =\left[K_{i}, K_{j}\right]\left(\varphi_{v}, \varphi_{v}, \varphi_{v}\right) \\
& =-8 d \varphi_{v}\left(K_{i}\left(\varphi_{v}\right), K_{j}\left(\varphi_{v}\right)\right) ; \quad i, j=1,2 \ldots n, \quad v \in V .
\end{aligned}
$$

The $k_{i}$ 's are vertically independent so, for each $v \in V$, the vector fields $K_{i}\left(\varphi_{v}\right)$ are linearly independent over $F(M)$ : Eq. (4.50), therefore, implies that each $\varphi_{v}$ is closed (note that the section $\left\{\left(m, \alpha_{(m)}\right)\right\} \subset T^{*} M$ must avoid $U$ since the vector fields $K_{i}(\alpha)$ are linearly dependent and, in fact, proportional). Thus $\Phi$ is a local complete solution of the Hamilton-Jacobi equation. If, for each $K_{i}, u_{i}$ and $w_{i}$ are defined as in Proposition 3.4 then:

$$
\mathscr{L}_{T}\left(G-\left(w_{i / u_{\imath}}\right) \cdot K_{i}\right)=0 ; \quad i=1,2 \ldots n .
$$

For each $v \in V$, the $n-1$ vector, fields $\left(G-\left(w_{i / u_{i}}\right) \cdot K_{i}\right)\left(\varphi_{v}\right)$ are independent over $F(M)$ so all the solutions $\beta \in \wedge_{1}(M)$ of the equation:

$$
\left(G-\left(w_{i / u_{u}}\right) \cdot K_{i}\right)\left(\varphi_{v}\right)(\beta)=0
$$

are proportional (for fixed $v$ ); $\alpha$ is one solution and, by Eq. $(4.51), \mathscr{L}_{T}\left(\varphi_{v}\right)$ is another. Hence $\mathscr{L}_{T}\left(\varphi_{v}\right)$ is proportional to $\alpha$, that is:

$$
\mathscr{L}_{T}\left(\perp \varphi_{v}\right)=0 ; \quad v \in V
$$

where $\perp$ is the projection relative to $(T, \alpha)$.

For completeness, I add a simplified proof of Eisenhart's theorem:

Corollary (Eisenhart's theorem [7]). If $(M, g)$ admits $n-1$ valence-2 Killing tensors $K_{1}, K_{2} \ldots K_{n-1}$ such that:

1) $K_{1}, K_{2} \ldots K_{n-1}, G$ are linearly independent over $F(M)$.

2) $K_{1}, K_{2} \ldots K_{n-1}$ have $n$ orthogonal closed common eigenforms

$$
\alpha_{1}, \alpha_{2} \ldots \alpha_{n} \in \wedge_{1}(M)
$$

then $\alpha_{1}, \alpha_{2} \ldots \alpha_{n}$ define (locally) an orthogonal separable coordinate system on $M$.

Proof. Put $K_{n}=G$ and $T_{i}=G\left(\alpha_{i}\right) / G\left(\alpha_{i}, \alpha_{i}\right)$. It is not hard to see that the functions $k_{1}, k_{2} \ldots k_{n}$ must be vertically independent. Define a local trivialization $\Phi: M \times V \subset M \times \mathbb{R}^{n} \rightarrow T^{*} M^{+}$as above and deduce that:

$$
\mathscr{L}_{T_{i}}\left(\perp_{i} \varphi_{v}\right)=0 ; \quad v \in V ; \quad i=1,2 \ldots n
$$

where $\perp_{i}$ is the projection relative to $\left(T_{i}, \alpha_{i}\right)$. The proof can be completed by noting that the closure of each $\varphi_{v}$ follows directly from Eq. (4.54).

With the exception of Proposition 4.3 and Theorem 4.1, all these results still hold true with the substitutions: "separable system for the null energy surface" for "separable system", "conformal Killing vector" for "Killing vector", "conformal Killing tensor" for "Killing tensor" etc. The argument breaks down at the point where case $3(([A, G],[B, G])$ is a non-trivial Killing pair $)$ is excluded. In 
place of Eq. (4.38), one obtains the tensor identity:

$$
[A, G] \cap\left[A-\frac{1}{2} B \cap B, G\right]+[B, G] \cap[B, G] \cap A=G \cap F
$$

for some $F \in S^{4}(M)$. I can see no immediate way of deducing that $A$ and $B$ must be conformal Killing tensors or that $[B, G]$ is a factor of $[A, G]$ and I must leave open the question of whether or not there exist separable systems for the null energy surface which give rise to non-trivial conformal Killing pairs.

\section{§ 5. Some Naïve Remarks on Global Problems}

The simplest way to define a global separable system $(T, \alpha)$ would be to require that there existed a complete global solution of the Hamilton-Jacobi equation which separated everywhere with respect to $(T, \alpha)$ in the sense of Eq. (4.2). However, as was remarked in $\S 4$, a solution of the Hamilton-Jacobi equation is, in effect, a hypersurface orthogonal congruence of geodesics and, in general, such congruences are not well behaved globally (they encounter caustics) so complete global solutions cannot be assumed to exist. Instead, I shall call $(T, \alpha)$ a (global) separable system if it is everywhere a (local) separable system in the sense of $\S 4$. The question, which I pose but make no real attempt to answer, is this: given a separable system $(T, \alpha)$, the method of the previous section can be used to construct local Killing tensor and vector fields; under what circumstances will these local fields extend to global fields and define proper invariant submanifolds of $T^{*} M$ ? It is conjectured that this question is closely related to the global problem of separating the wave equations on $M$. The following illustrates possible ways in which the local considerations of the previous section can break down in the large.

Examples. 1) Consider the flat Möbius band obtained by identifying the lines:

$$
x^{1}+x^{2}= \pm 1
$$

in $\mathbb{R}^{2}$ (with a reversal of orientation). Define the vector field $T$ and the closed 1 -form $\alpha$ according to:

$$
\begin{aligned}
& T=u \cdot\left(X_{1}-X_{2}\right)+X_{2} ; \quad X_{1}=\frac{\partial}{\partial x^{1}}, X_{2}=\frac{\partial}{\partial x^{2}} \\
& \alpha=d x^{1}+d x^{2}
\end{aligned}
$$

where $u$ is any real function such that $d u$ is parallel to $\alpha$ and $u=\frac{1}{2}$ on the line of identification [for example, $\left.u=-\frac{1}{2} \cos \left(\pi\left(x^{1}+x^{2}\right)\right)\right]$. By the third corollary to Proposition $4.1,(T, \alpha)$ is everywhere a local separable system of type II. However the local Killing vectors generated by $(T, \alpha)$ are linear combinations (over $\mathbb{R}$ ) of $X_{1}$ and $X_{2}$; except for special choices of the initial surface, these do not extend to global Killing vector fields $\left(X_{1}\right.$ and $X_{2}$ are interchanged on the line of identification).

In this example the local constants of the motion do, in fact, define invariant submanifolds of the cotangent bundle since the corresponding local Killing vector fields are simply double valued in the large. This is not a feature which could be expected in general. 
2) Consider the metric on $\mathbb{R}^{n}$ with contravariant components:

$$
G^{\mathrm{a} b}=\frac{1}{u}\left[\begin{array}{ccccc}
v & 0 & 0 & \ldots & 0 \\
0 & w_{22} w_{23} & \ldots & w_{2 n} \\
0 & w_{32} w_{33} & \ldots & w_{3 n} \\
0 & w_{n 2} w_{n 3} & \ldots & w_{n n}
\end{array}\right]
$$

where:

$$
\begin{aligned}
& u=u_{1}\left(x^{1}\right)+u_{2}\left(x^{2}, \ldots x^{n}\right), \\
& v=v\left(x^{1}\right), \\
& w_{\mathrm{ij}}=w_{\mathrm{ij}}\left(x^{2}, \ldots x^{n}\right) ; \quad \mathrm{i}, \mathrm{j}=2,3 \ldots n,
\end{aligned}
$$

are functions such that:

$$
\mathrm{G}^{\mathfrak{a} \mathfrak{b}}=\delta^{\mathfrak{a} \mathfrak{b}}
$$

outside the neighbourhood:

$$
\left\{\left(x^{1}, x^{2} \ldots x^{n}\right) ;-\frac{1}{2}<x^{1}<\frac{1}{2}\right\}
$$

of the origin. The Riemannian manifold $(M, g)$ is constructed by identifying the planes $x^{1}= \pm 1$ according to:

$$
\left(1, x^{2} \ldots x^{n}\right)=\left(-1, x^{2}+r, x^{3} \ldots x^{n}\right) ; \quad r \in \mathbb{R} .
$$

Put $T=\frac{\partial}{\partial x^{1}}$ and $\alpha=d x^{1}$. Clearly $(T, \alpha)$ is everywhere a local type I separable system. Taking as initial surface:

$$
S=\left\{\left(0, x^{2}, x^{3} \ldots x^{n}\right)\right\}
$$

the construction described in $\S 4$ yields a Killing tensor $A$ defined in some neighbourhood $U$ of $S$. However, it is not possible to extend $A$ to a global Killing tensor field by Lie propagating along the integral curves of $T$. For example, the integral curve through the origin intersects $S$ at the points $(0, m r, 0, \ldots 0)$, $m=\ldots-1,0,1,2 \ldots$, and at each of these points $A$ has a different value (in the naïve coordinate dependent sense).

For a type II separable system there does exist one clear sufficient condition for the constants of the motion to be well behaved globally, that is that the Lie algebras of local Killing vectors from which the system is constructed should generate a group of (global) isometries. In the first example above the two Killing vectors $X_{1}$ and $X_{2}$ do not generate isometries. The separation properties of a Riemannian manifold with an abelian isometry group are well understood.

For type I orthogonal separable systems one can also give one obvious global criterion, that is that $\alpha=d x$ should be exact and that $x$ should have no critical points. In fact, provided that $T$ is sufficiently well behaved near the critical sets of $x$ and provided these sets are not too large, the Killing tensors $A_{t}$ will be well behaved globally. For instance, this will certainly be the case if $x: M \rightarrow \mathbb{R}$ has a 
local coordinate form:

$$
x=x(0) \pm\left(x^{1}\right)^{2} \pm\left(x^{2}\right)^{2} \pm \ldots \pm\left(x^{m}\right)^{2} ; \quad m \geqq 2
$$

near its critical points. The proof is based on a simple geometrical argument which hinges on the constancy of the functions $a_{t}: T^{*} M \rightarrow \mathbb{R}$ along the geodesics near the critical points of $x$.

All that can really be concluded from these remarks is that a full answer to the question will depend on details of the relationship between $T, \alpha$, and the global structure of the base manifold.

\section{$\S 6$. Type $\{2,2\}$ Space-Times}

In this section, I shall illustrate some of my work by applying it to a certain class of algebraically special vacuum solutions of Einstein's equations; no new results will be obtained, but some old ones may be illuminated. For computational efficiency, I shall adopt the Battelle convention according to which spinor and tensor fields are labelled by abstract indices appropriate to their valences [22].

Summarizing some results of Hughston, Penrose, Sommers and Walker [13, $31]$ : in any type $\{2,2\}$ vacuum space-time $\left(M, g_{a b}\right)$ with principal spinors $o^{A}$ and $t^{A}$ (normalized so that $o_{A}{ }^{A}=1$ ) there exist complex valued functions $\alpha, \psi: \mathrm{M} \rightarrow \nsubseteq$ such that:

1) $\Psi_{A B C D}=\psi O_{(A} O_{B} l_{C} l_{D)}$ is the Weyl spinor.

2) $X_{A B}=\psi^{-1 / 3} o_{(A} l_{B)}$ satisfies the twistor equation:

$\nabla_{(A}^{A^{\prime}} X_{B C)}=0$.

3) $\phi_{A B}=\psi^{2 / 3} o_{(A} l_{B)}$ satisfies Maxwell's equation:

$\nabla_{A^{\prime}}^{A} \phi_{A B}=0$.

4) $\psi_{A B C D}=\alpha \Psi_{A B C D}$ satisfies the Einstein-Maxwell-Bianchi identity:

$\nabla_{A^{\prime}}^{A} \psi_{A B C D}=\nabla_{(B}^{B^{\prime}} \Phi_{C D) A^{\prime} B^{\prime}}$

where $\Phi_{C D A^{\prime} B^{\prime}}=\phi_{C D} \phi_{A^{\prime} B^{\prime}}$.

If $Z^{a}$ is any Killing vector then:

$$
\begin{aligned}
0= & \mathscr{L}_{Z}\left(C_{a b c d}\right)=\left(\mathscr{L}_{Z}\left(\Psi_{A B C D}\right)\right) \varepsilon_{A^{\prime} B^{\prime}} \varepsilon_{C^{\prime} D^{\prime}} \\
& +\left(\mathscr{L}_{Z}\left(\bar{\Psi}_{A^{\prime} B^{\prime} C^{\prime} D^{\prime}}\right)\right) \varepsilon_{A B^{\prime}} \varepsilon_{C D}
\end{aligned}
$$

where $C_{a b c d}$ is the Weyl tensor (the Lie derivative of a spinor with respect to a Killing vector has been defined by Geroch; the operation commutes with complex conjugation, raising and lowering of indices, contraction and covariant differentiation and it reduces to the normal Lie derivative on tensors [10, 27]). Equation (6.4) and the symmetry of $\Psi_{A B C D}$ give:

$$
\mathscr{L}_{Z}\left(\Psi_{A B C D}\right)=0 .
$$


Hence:

$$
\begin{gathered}
Z^{a} \nabla_{a}(\psi)=0, \\
\mathscr{L}_{Z}\left(o_{(A} l_{B)}\right)=0, \\
\mathscr{L}_{Z}\left(X_{A B}\right)=0, \\
\mathscr{L}_{Z}\left(\phi_{A B}\right)=0 .
\end{gathered}
$$

It has been shown by Hughston and Sommers [13] that the complex vector:

$$
X_{a}=\nabla_{A^{\prime}}^{B}, X_{B A}=\phi_{A B} \nabla_{A^{\prime}}^{B} \psi^{-1}
$$

satisfies the Killing equation:

$$
\nabla_{(a} \mathrm{X}_{b)}=0 \text {. }
$$

Also, by virtue of Eq. (6.1), $P_{a b}=X_{A B} \bar{X}_{A^{\prime} B^{\prime}}$ is a trace-free conformal Killing tensor. The condition that $P_{a b}$ be the trace-free part of a Killing tensor $C_{a b}$ is that there should exist a real function $k \in F(M)$ such that:

$$
\nabla_{(a}\left(P_{b c)}+\frac{1}{4} k \cdot g_{b c)}\right)=0
$$

that is, such that:

$$
\frac{3}{4} \nabla_{a} k=\Phi_{a b} \nabla^{b}(\psi \bar{\psi})^{-1}=-\frac{1}{4}\left(\bar{\psi}^{-1} \nabla_{a} \alpha=\psi^{-1} \nabla_{a} \bar{\alpha}\right) .
$$

When the integrability condition for this equation is satisfied (as it is in the Kerr metric) $\alpha$ is a function only of $\psi$ and $\bar{\psi}$ and so, for any Killing vector $Z^{a}$ :

$$
Z^{a} \nabla_{a} \alpha=0=Z^{a} \nabla_{a} \bar{\alpha} ; \quad Z^{a} \nabla_{a} k=0 .
$$

In the following, I shall assume that the integrability condition is satisfied, so that there does exist a Killing tensor $C_{a b}$ (with trace free part $P_{a b}$ ).

Combining Eqs. (6.14) and (6.8), one obtains $\mathscr{L}_{Z}\left(C_{a b}\right)=0$ for any Killing vector $Z^{a}$. Further, from Eqs. (6.8) and (6.10), $\mathscr{L}_{Z}\left(X^{a}\right)=0$. Now, both the real and imaginary parts of $X^{a}$ must be Killing vectors so, except in the degenerate case when $X^{a}$ and $\bar{X}^{a}$ are dependent, $M$ admits three distinct Killing tensors:

$$
\begin{aligned}
& K_{1}^{a b}=C^{a b}, \\
& K_{2}^{a b}=\left(X^{(a}+\bar{X}^{(a}\right)\left(X^{b)}+\bar{X}^{b)}\right), \\
& K_{3}^{a b}=\left(X^{(a}-\bar{X}^{(a}\right)\left(X^{b)}-\bar{X}^{b)}\right) .
\end{aligned}
$$

These can be shown to be independent [31]. In the degenerate case, a third Killing tensor is defined by:

$$
K_{3}^{a b}=Y^{(a} Y^{b)}
$$

where $Y^{a}$ is the Killing vector:

$$
Y^{a}=C^{a b}\left(X_{b}+\bar{X}_{b}\right) \text {. }
$$

In both cases, $K_{1}^{a b}, K_{2}^{a b}$, and $K_{3}^{a b}$ commute.

Now, from Eqs. (6.1) and (6.12):

$$
\left.C_{a b}=u \cdot L_{(a} N_{b)}+v \cdot M_{(a} \bar{M}_{b}\right)
$$


where:

$$
\begin{aligned}
u & =\frac{1}{2}\left((\psi \bar{\psi})^{-1 / 3}+k\right), \quad v=\frac{1}{2}\left((\psi \bar{\psi})^{-1 / 3}-k\right), \\
L^{a} & =O^{A} \bar{O}^{A^{\prime}}, \quad N^{a}=l^{A} \bar{l}^{A^{\prime}}, \quad M^{a}=O^{A} \bar{l}^{A^{\prime}} .
\end{aligned}
$$

Also, from Eqs. (6.6) and (6.14), $Z^{a} \nabla_{a} u=0$ for any Killing vector $Z^{a}$, while transvecting the Killing equation for $C_{a b}$ with $L^{a} L^{b} N^{c}$ and with $N^{a} N^{b} L^{c}$ gives:

$$
L^{a} \nabla_{a} u=0=N^{a} \nabla_{a} u .
$$

Thus the exact 1-form $\nabla_{a} u$ is a common eigenform of $K_{1}^{a b}, K_{2}^{a b}$ and $K_{3}^{a b}$. By applying Theorem 4.2, $\left(T^{a}, \nabla_{a} u\right)$ can be seen to be an orthogonal separable system, where:

$$
T^{a}=\nabla^{a} u /\left(\nabla^{b} u\right)\left(\nabla_{b} u\right) .
$$

The canonical example of a type $\{2,2\}$ vacuum space-time for which Eq. (6.13) is integrable is the Kerr solution. In the standard Boyer-Lindquist coordinates $\{t, r, \theta, \phi\}$, the metric has the form [3]:

$$
g_{\mathfrak{a b}}=\left[\begin{array}{cccc}
1-\frac{2 m r}{R^{2}} & 0 & 0 & \frac{2 m a r \cdot \sin ^{2} \theta}{R^{2}} \\
0 & \frac{-R^{2}}{r^{2}-2 m r+a^{2}} & 0 & 0 \\
0 & 0 & -R^{2} & 0 \\
\frac{2 m a r \cdot \sin ^{2} \theta}{R^{2}} & 0 & 0 & -\sin ^{2} \theta\left(r^{2}+a^{2}+\frac{2 m a^{2} r \cdot \sin ^{2} \theta}{R^{2}}\right)
\end{array}\right]
$$

where $R^{2}=r^{2}+a^{2} \cdot \cos ^{2} \theta$. In an alternative coordinate system (used by Walker and Penrose [31]) the metric components are:

$$
\left[\begin{array}{cccc}
1-\frac{2 m r^{\prime}}{R^{\prime 2}} & -1 & 0 & \frac{2 m a r^{\prime} \cdot \sin ^{2} \theta^{\prime}}{R^{\prime 2}} \\
-1 & 0 & 0 & a \cdot \sin ^{2} \theta^{\prime} \\
0 & 0 & -R^{\prime 2} & 0 \\
\frac{2 m r^{\prime} a \cdot \sin ^{2} \theta^{\prime}}{R^{\prime 2}} & a \cdot \sin ^{2} \theta^{\prime} & 0 & -\sin ^{2} \theta^{\prime}\left(r^{\prime 2}+a^{2}+\frac{2 m a^{2} r^{\prime} \cdot \sin ^{2} \theta^{\prime}}{R^{\prime 2}}\right)
\end{array}\right]
$$

the two systems being related by:

$$
\begin{aligned}
d t & =d t^{\prime}-\left(r^{2}+a^{2}-2 m r\right)^{-1} \cdot\left(r^{2}+a^{2}\right) \cdot d r^{\prime} \\
d r & =d r^{\prime} \\
d \theta & =d \theta^{\prime} \\
d \phi & =d \phi^{\prime}-a\left(r^{2}+a^{2}-2 m r\right) \cdot d r .
\end{aligned}
$$

Borrowing from the calculations of Walker and Penrose [31] (and the corrections of Hughston, Penrose, Sommers, and Walker [13]):

$$
\begin{aligned}
& \psi=6 m(r-i a \cdot \cos \theta)^{-3}, \\
& k=-(6 m)^{-2 / 3}\left(r^{2}-a^{2} \cos ^{2} \theta\right),
\end{aligned}
$$


so that, for this solution, $u=(6 m)^{-2 / 3} \cdot a^{2} \cdot \cos ^{2} \theta$ : the $\theta$ coordinate is thus seen to be separable. This result was, of course, established some time ago by Carter [5]; however, one advantage of the present somewhat circuitous proof is that the global nature of the separation is a little more transparent.

The ignorable coordinates, $t$ and $\phi$, are trivially separable, so it is a corollary of Proposition 4.1 that the Boyer-Lindquist coordinate system is separable. Explicitly, a complete separable solution:

$$
\Psi:\left(t, r, \theta, \phi, v_{1}, v_{2}, v_{3}, v_{4}\right) \mapsto\left(t, r, \theta, \phi, \psi_{1}, \psi_{2}, \psi_{3}, \psi_{4}\right) \in T^{*} M
$$

is given by $[2,5]$ :

$$
\begin{aligned}
\psi_{1}= & v_{1} \\
\psi_{2}= & \left\{r\left(r\left(r^{2}+a^{2}\right)+2 a^{2} m\right) \cdot v_{1}^{2}-4 a m r \cdot v_{1} \cdot v_{2}-\left(r^{2}-2 m r\right) \cdot v_{4}^{2}\right. \\
& \left.-\left(r^{2}+a^{2}-2 m r\right)\left(r^{2} \cdot v_{3}+v_{2}\right)\right\}^{1 / 2} \cdot\left(r^{2}+a^{2}-2 m r\right)^{-1} \\
\psi_{3}= & \left\{v_{2}+a^{2}\left(v_{1}^{2}-v_{3}^{2}\right) \cdot \cos ^{2} \theta-v_{4}^{2} \cdot \cot ^{2} \theta\right\}^{1 / 2} \\
\psi_{4}= & v_{4} .
\end{aligned}
$$

The constants of the motion constructed from the functions:

$$
k_{i}: v \mapsto v_{i}
$$

(as in Theorem 2.1) are those associated with, respectively, the Killing vector $\frac{\partial}{\partial}$, the Killing tensor $C^{a b}$ (otherwise known as Carter's fourth constant), the $\partial t$

metric and the Killing vector $\frac{\partial}{\partial \phi}$.

The conformal rigidities of $\frac{\partial}{\partial \theta}$ and $\frac{\partial}{\partial r}$ are seen from Eq. (6.24) to be 3 and 4 respectively, while from Eq. (6.30) it is clear that the numbers of linearly independent [over $F(M)$ ] Killing tensors generated by the orthogonal separable systems $\left(\frac{\partial}{\partial \theta}, d \theta\right)$ and $\left(\frac{\partial}{\partial r}, d r\right)$ are, respectively, 4 and 5 ; in each case the metric is included. This is in agreement with Proposition 4.2.

Finally, the coordinates $\left(t^{\prime}, r^{\prime}, \theta^{\prime}, \phi^{\prime}\right)$ are also separable. The (non-orthogonal) separable system $\left(\frac{\partial}{\partial x^{\prime}}, d r^{\prime}\right)$ is obtained from from the orthogonal separable system $\left(\frac{\partial}{\partial r}, d r\right)$ by two transformations of the type described in the second corollary to Proposition 4.1.

Acknowledgements. I thank Lane Hughston, Paul Sommers, Martin Walker, and Yavuz Nutku for enlightening conversations. I thank, especially, Felix Pirani for many suggestions and tireless criticism. 


\section{References}

1. Abraham, R., Marsden,J.E.: Foundations of mechanics. Reading, Mass.: Benjamin 1967

2. Bardeen, J.M.: Timelike and null geodesics in the Kerr metric, 1972. Les Houches Summer School, pp. 215-239. London-New-York-Paris: Gordon and Breach 1973

3. Boyer, R.H., Lindquist, R. W.: J. Math. Phys. 8, 265 (1967)

4. Burgatti, P.: R. Acad. dei Lincei (Roma) 20, 108 (1911)

5. Carter, B.: Phys. Rev. 174, 1559 (1968); and: Black Hole Equilibrium States. 1972, Les Houches Summer School, pp. 57-214. London-New-York-Paris: Gordon and Breach 1973

6. Dall'Acqua,F.A.: Math. Ann. 66, 398 (1908); and: Rend. di Palermo 33, 341 (1912)

7. Eisenhart, L.P.: Ann. Math. 35, 284 (1934)

8. Galgani,L., Scotti, A.: Nuovo Cimento 2, 189 (1972)

9. Geroch, R.P.: J. Math. Phys. 11, 1955 (1970)

10. Geroch,R.P.: Special topics in particle physics: mimeographed lecture notes: Chicago 1971

11. Hauser, I., Malhiot, R. J.: J. Math. Phys. (to appear) (1975)

12. Herman, R.: Differential geometry and the calculus of variations. New York: Academic Press 1968

13. Hughston, L.P., Penrose, R., Sommers,P., Walker, M.: Commun. math. Phys. 27, 303 (1972); Hughston, L.P., Sommers, P.: Commun. math. Phys. 32, 147 (1973); and: Commun. math. Phys. 33, 129 (1973)

14. Iarov-Iarovoi, M. S.: J. Appl. Math. Mech. 27, 1499 (1964)

15. Kerr, R. P.: Phys. Rev. Lett. 11, 237 (1963)

16. Kostant, B.: Symplectic spinors. Symposia Math. to appear

17. Levi-Civita, T.: Math. Ann. 49, 383 (1904)

18. Liouville, J.: J. de Math. 11, 345 (1846)

19. Loomis, L.H., Sternberg, S.: Advanced calculus. Reading, Mass.: Addison Wesley 1968

20. Nijenhuis, A.: Nederl. Akad. Wetensch. Proc. Ser. A 58, 390 (1955)

21. Onofri, E., Pauri, M.: J. Math. Phys. 14, 1106 (1973)

22. Penrose,R.: Structure of space-time. In: De Witt,C., Wheeler,J.A. (Eds.): Battelle rencontres, pp. 121-235. New York: Benjamin 1968

23. Pirani, F. A.E.: Capt. 8 of A. Trautman's lectures. 1964 Brandeis Summer Institute, pp. 201-227. Englewood Cliffs, N. J.: Prentice-Hall 1965

24. Ricci, G., Levi-Civita, T.: Math. Ann. 54, 178 (1901)

25. Stäckel, P.: Math. Ann. 42, 537 (1893); and: C. R. Acad. Sci. Paris 121, 489 (1895)

26. Serre, J.-P.: Lie groups and Lie algebras. New-York-Amsterdam: Benjamin 1965

27. Sommers, P.: J. Math. Phys. 14, 787 (1973)

28. Sommers, P.: Killing tensors and type $\{2,2\}$ space-times. Ph. D. Austin 1973

29. Stewart,J.M., Walker, M.: Perturbations of space-times in general relativity. Preprint, MaxPlanck-Institut, Munich 1973

30. Teukolsky, S. A.: Perturbations of rotating black holes, I. Press, W. A., Teukolsky, S. A.: Perturbations of rotating black holes, II. Preprint, California Institute of Technology 1973

31. Walker, M., Penrose, R.: Commun. Math. Phys. 18, 265 (1970)

32. Walker, M.: Private communication

Communicated by J. Ehlers

N. M. J. Woodhouse

Department of Mathematics

University of London

King's College

London WC2R 2LS, U. K. 